\title{
Homogeneous Poisson Structures on Loop Spaces of Symmetric Spaces
}

\section{Doug PICKRELL}

Department of Mathematics, University of Arizona, Tucson, AZ, 85721, USA

E-mail: pickrell@math.arizona.edu

Received June 14, 2008, in final form September 27, 2008; Published online October 07, 2008

Original article is available at http://www.emis.de/journals/SIGMA/2008/069/

\begin{abstract}
This paper is a sequel to [Caine A., Pickrell D., Int. Math. Res. Not., to appear, arXiv:0710.4484], where we studied the Hamiltonian systems which arise from the Evens$\mathrm{Lu}$ construction of homogeneous Poisson structures on both compact and noncompact type symmetric spaces. In this paper we consider loop space analogues. Many of the results extend in a relatively routine way to the loop space setting, but new issues emerge. The main point of this paper is to spell out the meaning of the results, especially in the $S U(2)$ case. Applications include integral formulas and factorizations for Toeplitz determinants.
\end{abstract}

Key words: Poisson structure; loop space; symmetric space; Toeplitz determinant

2000 Mathematics Subject Classification: 22E67; 53D17; 53D20

\section{Introduction}

The first purpose of this paper is to generalize the framework in [3] to loop spaces. This generalization is straightforward, using the fundamental insight of Kac and Moody that finite dimensional complex semisimple Lie algebras and (centrally extended) loop algebras fit into the common framework of Kac-Moody Lie algebras.

Suppose that $\dot{X}$ is a simply connected compact symmetric space with a fixed basepoint. From this, as we will more fully explain in Sections 2 and 3, we obtain a diagram of groups

$$
G_{0}=\widehat{L} \dot{G}_{0} \nearrow^{\nwarrow} \begin{gathered}
G=\widehat{L} \dot{G} \\
K=\widehat{L} \dot{K}
\end{gathered} \nearrow U=\widehat{L} \dot{U}
$$

where $\dot{U}$ is the universal covering of the identity component of the isometry group of $\dot{X}, \dot{X} \simeq$ $\dot{U} / \dot{K}, \dot{G}$ is the complexification of $\dot{U}, \dot{X}_{0}=\dot{G}_{0} / \dot{K}$ is the noncompact type symmetric space dual to $\dot{X}, L \dot{G}$ denotes the loop group of $\dot{G}, \widehat{L} \dot{G}$ denotes a Kac-Moody extension, and so on. This diagram is a prolongation of diagram (0.1) in [3] (which is embedded in (1.1) by considering constant loops).

We also obtain a diagram of equivariant totally geodesic (Cartan) embeddings of symmetric spaces:

$$
\begin{array}{cccccc}
L(\dot{U} / \dot{K}) & \stackrel{\phi}{\rightarrow} & \widehat{L} \dot{U} & & \\
\downarrow & & \downarrow & & \\
\tilde{L} \dot{G} / \tilde{L} \dot{G}_{0} & \stackrel{\phi}{\rightarrow} & \widehat{L} \dot{G} & \stackrel{\psi}{ } & & \tilde{L} \dot{G} / \tilde{L} \dot{U} \\
& & \uparrow & & \uparrow \\
& & \widehat{L} \dot{G}_{0} & \stackrel{\psi}{\leftarrow} & L\left(\dot{G}_{0} / \dot{K}\right)
\end{array}
$$

\footnotetext{
${ }^{\star}$ This paper is a contribution to the Special Issue on Kac-Moody Algebras and Applications. The full collection is available at http://www.emis.de/journals/SIGMA/Kac-Moody_algebras.html
} 
This is a prolongation of diagram (0.2) in [3].

Let $\Theta$ denote the involution corresponding to the pair $(\dot{U}, \dot{K})$. We consider one additional ingredient: a triangular decomposition

$$
\dot{\mathfrak{g}}=\dot{\mathfrak{n}}^{-} \oplus \dot{\mathfrak{h}} \oplus \dot{\mathfrak{n}}^{+}
$$

which is $\Theta$-stable and for which $\dot{\mathfrak{t}}_{0}=\dot{\mathfrak{h}} \cap \dot{\mathfrak{k}}$ is maximal abelian in $\dot{\mathfrak{k}}$. There is a corresponding Kac-Moody triangular decomposition

$$
\widehat{L}_{\text {pol }} \dot{\mathfrak{g}}=\left(\bigoplus_{n<0} \dot{\mathfrak{g}} z^{n} \oplus \dot{\mathfrak{n}}^{-}\right) \oplus \mathfrak{h} \oplus\left(\dot{\mathfrak{n}}^{+} \oplus \bigoplus_{n>0} \mathfrak{g} z^{n}\right)
$$

extending (1.3).

This data determines standard Poisson Lie group structures, denoted $\pi_{U}$ and $\pi_{G_{0}}$, for the groups $U=\widehat{L} \dot{U}$ and $G_{0}=\widehat{L} \dot{G}_{0}$, respectively. By a general construction of Evens and Lu [4], the symmetric spaces $X=L \dot{X}$ and $X_{0}=L \dot{X}_{0}$ acquire Poisson structures $\Pi_{X}$ and $\Pi_{X_{0}}$, respectively, which are homogeneous for the respective actions of the Poisson Lie groups $\left(U, \pi_{U}\right)$ and $\left(G_{0}, \pi_{G_{0}}\right)$. These spaces are infinite dimensional, and there are many subtleties associated with Poisson structures in infinite dimensions (see [8]). Consequently in this paper we will always display explicit decompositions and formulas, and we will avoid any appeal to general theory (for "symplectic foliations", for example).

The plan of this paper is the following. In Section 2 we introduce notation and recall some well-known facts concerning loop algebras and groups.

In Section 3 we consider the case when $\dot{X}$ is an irreducible type I space. All of the results of Sections 1-4 of [3] generalize in a relatively straightforward way to the loop context roughly outlined above. The basic result is that $\Pi_{X_{0}}$ has just one type of symplectic leaf, this leaf is Hamiltonian with respect to the natural action of $T_{0}$, there are relatively explicit formulas for this Hamiltonian system, and in a natural way, this system is isomorphic to the generic Hamiltonian system for $\Pi_{X}$. Although this system is infinite dimensional, a heuristic application of the Duistermaat-Heckman exact stationary phase theorem to this system suggests some remarkable integral formulas. This is discussed in Section 7 of [9]. These formulas remain conjectural.

In Section 4 I have attempted to do some calculations in the $\dot{X}=S^{2}$ case. The formulas are complicated; I included them to give the reader a concrete feeling for the subject.

In Section 5, and Appendix A, we consider the group case. Again, the results of Sections 1-4 of [3] generalize in a straightforward way. However significant issues emerge when we try to generalize the results of Section 5 of [3]. In the finite dimensional context of $\dot{X}=\dot{K}$, the (negative of the) standard Poisson Lie group structure $\left(\dot{K}, \pi_{\dot{K}}\right)$ is isomorphic to $\left(\dot{X}, \Pi_{\dot{X}}\right)$, by left translation by a representative for the longest Weyl group element. In the loop context the Poisson Lie group and Evens-Lu structures are fundamentally different: the symplectic leaves for $\pi_{K}$ (essentially Bruhat cells) are finite dimensional, whereas the symplectic leaves for $\Pi_{X}$ (essentially Birkhoff strata) are finite codimensional. In finite dimensions Lu has completely factored the symplectic leaves. Lu's results, as formulated in [7] in terms of $\pi_{K}$, do generalize in a relatively straightforward way to the loop context. Some details of this generalization are worked out in Appendix A, where we have extended this to the larger category of symmetrizable Kac-Moody algebras.

The basic question is whether the Hamiltonian systems for $\Pi_{X}$, in this infinite dimensional context, are solvable (in a number of senses). In Sections 5-7 we show that there is a natural way to conjecturally reformulate and extend Lu's results to suggest that the generic symplectic leaves are integrable. However we have not succeeded in fully proving this conjecture: while we can factor the momentum mapping, and the Haar measure relevant for Theorem 1 below, we have not shown that the symplectic form $\Pi_{X}^{-1}$ factors. 
In Section 6 we spell out the meaning of the results in Section 5 when $\dot{K}=S U(2)$. One consequence is the following integral formula.

Theorem 1. Given $x_{j} \in \mathbb{C}$, let

$$
B\left(\sum_{j=1}^{n} x_{j} z^{j}\right)=\left(\begin{array}{ccccc}
x_{n} & 0 & & \ldots & 0 \\
x_{n-1} & x_{n} & 0 & \ldots & 0 \\
\vdots & & \ddots & \ddots & \vdots \\
x_{2} & \ldots & x_{n-1} & x_{n} & 0 \\
x_{1} & x_{2} & \ldots & x_{n-1} & x_{n}
\end{array}\right) .
$$

Then

$$
\begin{aligned}
& \int \prod_{l=0}^{n-1} \operatorname{det}\left(1+B\left(\sum_{j=1}^{n-l} x_{l+j} z^{j}\right)\right) B\left(\sum_{j=1}^{n-l}\left(x_{l+j} z^{j}\right)^{*}\right)^{-p_{l}} d \lambda\left(x_{1}, \ldots, x_{n}\right) \\
& \quad=\pi^{n} \frac{1}{\left(p_{1}-1\right)} \frac{1}{\left(2 p_{1}+p_{2}-3\right)} \cdots \frac{1}{\left(n p_{1}+(n-1) p_{2}+\cdots+p_{n}-(2 n-1)\right)} .
\end{aligned}
$$

In particular, if we write $B_{n}(x)$ for the matrix (1.4), for a general power series $x=\sum x_{j} z^{j}$, then

$$
\frac{1}{\operatorname{det}\left(1+B_{n}(x) B_{n}(x)^{*}\right)^{p}} d \lambda\left(x_{1}, \ldots, x_{n}\right)
$$

is a finite measure if and only if $p>2-1 / n$.

This result is important because it determines the critical exponents for the integrands in (1.5) exactly, whereas I am not aware of any other way to even estimate these exponents in a useful way. The relevance of this to the theory of conformally invariant measures, where one must understand the limit as $n \rightarrow \infty$, is described in [10].

In Section 7 we consider the question of global solvability of the symplectic leaves, in the $S U(2)$ case. A consequence of the global factorization of the momentum mapping is the following illustrative statement about block Toeplitz operators.

Theorem 2. Given complex numbers $\eta_{j}, \chi_{j}, \zeta_{j}$, let $g: S^{1} \rightarrow S U(2)$ be the product of $S U(2)$ loops

$$
\begin{aligned}
& a\left(\eta_{0}\right)\left(\begin{array}{cc}
1 & \eta_{0} \\
-\bar{\eta}_{0} & 1
\end{array}\right) \cdots a\left(\eta_{n}\right)\left(\begin{array}{cc}
1 & \eta_{n} z^{n} \\
-\bar{\eta}_{n} z^{-n} & 1
\end{array}\right)\left(\begin{array}{cc}
e^{\sum \chi_{j} z^{j}} & 0 \\
0 & e^{-\sum \chi_{j} z^{j}}
\end{array}\right), \\
& a\left(\zeta_{n}\right)\left(\begin{array}{cc}
1 & \zeta_{n} z^{-n} \\
-\bar{\zeta}_{n} z^{n} & 1
\end{array}\right) \cdots a\left(\zeta_{1}\right)\left(\begin{array}{cc}
1 & \zeta_{1} z^{-1} \\
-\bar{\zeta}_{1} z & 1
\end{array}\right),
\end{aligned}
$$

where $a(\cdot)=\left(1+|\cdot|^{2}\right)^{-1 / 2}$ and $\chi_{-j}=-\bar{\chi}_{j}$. Let $A(g)$ denote the Toeplitz operator defined by the symbol $g$. Then

$$
\operatorname{det}\left(A(g) A(g)^{*}\right)=\prod_{j} a\left(\eta_{j}\right)^{2 j} a\left(\zeta_{j}\right)^{2 j} e^{-|j|\left|\chi_{j}\right|^{2}} .
$$

When $\eta$ and $\zeta$ vanish, this reduces to a well-known formula with a long history (e.g. see Theorem 7.1 of [12]).

In Section 7, because the $S U(2)$ loop space is infinite dimensional, it is necessary to take a limit as $n \rightarrow \infty$, so that the above product of loops is to be interpreted as an infinite factorization of a generic $g \in L S U(2)$. At a heuristic level, the invariant measures considered in [9] factor in these coordinates. The conjectural integral formulas in Section 7 of [9] (in the $S U(2)$ case) follow immediately from this product structure. However changing coordinates in infinite dimensions is nontrivial, and probabilistic analysis is required to justify this claim. 


\section{Loop groups}

In this section we recall how (extended) loop algebras fit into the framework of Kac-Moody Lie algebras. The relevant structure theory for loop groups is developed in [11], and for loop algebras in Chapter 7 of [5].

Let $\dot{U}$ denote a simply connected compact Lie group. To simplify the exposition, we will assume that $\dot{\mathfrak{u}}$ is a simple Lie algebra. Let $\dot{G}$ and $\dot{\mathfrak{g}}$ denote the complexifications, and fix a $\dot{\mathfrak{u}}$-compatible triangular decomposition

$$
\dot{\mathfrak{g}}=\dot{\mathfrak{n}}^{-} \oplus \dot{\mathfrak{h}} \oplus \dot{\mathfrak{n}}^{+} .
$$

We let $\langle\cdot, \cdot\rangle$ denote the unique $A d(\dot{G})$-invariant symmetric bilinear form such that (for the dual form) $\langle\theta, \theta\rangle=2$, where $\theta$ denotes the highest root for $\dot{\mathfrak{g}}$, i.e. $\langle\cdot, \cdot\rangle=\frac{1}{\dot{g}} \kappa$, where $\kappa$ denotes the Killing form, and $\dot{g}$ is the dual Coxeter number.

Let $\widehat{L} \dot{\mathfrak{g}}$ denote the real analytic completion of the untwisted affine Lie algebra corresponding to $\dot{\mathfrak{g}}$, with derivation included (the degree of smoothness of loops is essentially irrelevant for the purposes of this discussion; any fixed degree of Sobolev smoothness $s>1 / 2$ would work equally well). This is defined in the following way. We first consider the universal central extension of $L \dot{\mathfrak{g}}=C^{\omega}\left(S^{1}, \dot{\mathfrak{g}}\right)$,

$$
0 \rightarrow \mathbb{C} c \rightarrow \tilde{L} \dot{\mathfrak{g}} \rightarrow L \dot{\mathfrak{g}} \rightarrow 0
$$

As a vector space $\tilde{L} \dot{\mathfrak{g}}=L \dot{\mathfrak{g}} \oplus \mathbb{C} c$. In these coordinates, the $\tilde{L} \dot{\mathfrak{g}}$-bracket is given by

$$
\left[X+\lambda c, Y+\lambda^{\prime} c\right]_{\tilde{L} \dot{\mathfrak{g}}}=[X, Y]_{L \dot{\mathfrak{g}}}+\frac{i}{2 \pi} \int_{S^{1}}\langle X \wedge d Y\rangle c .
$$

Then $\widehat{L} \dot{\mathfrak{g}}=\mathbb{C} d \propto \tilde{L} \dot{\mathfrak{g}}$ (the semidirect sum), where the derivation $d$ acts by $d(X+\lambda c)=\frac{1}{i} \frac{d}{d \theta} X$, for $X \in L \dot{\mathfrak{g}}$. The algebra generated by $\dot{\mathfrak{u}}$-valued loops induces a central extension

$$
0 \rightarrow i \mathbb{R} c \rightarrow \tilde{L} \dot{\mathfrak{u}} \rightarrow L \dot{\mathfrak{u}} \rightarrow 0
$$

and a real form $\widehat{L} \dot{\mathfrak{u}}=i \mathbb{R} d \propto \tilde{L} \dot{\mathfrak{u}}$ for $\widehat{L} \dot{\mathfrak{g}}$. We identify $\dot{\mathfrak{g}}$ with the constant loops in $L \dot{\mathfrak{g}}$. Because the extension is trivial over $\dot{\mathfrak{g}}$, there are embeddings of Lie algebras

$$
\dot{\mathfrak{g}} \rightarrow \tilde{L} \dot{\mathfrak{g}} \rightarrow \widehat{L} \dot{\mathfrak{g}}
$$

The Lie algebra $\widehat{L} \dot{\mathfrak{g}}$ has a triangular decomposition

$$
\widehat{L} \dot{\mathfrak{g}}=\mathfrak{n}^{-} \oplus \mathfrak{h} \oplus \mathfrak{n}^{+},
$$

where

$$
\begin{aligned}
& \mathfrak{h}=\dot{\mathfrak{h}}+\mathbb{C} c+\mathbb{C} d, \\
& \mathfrak{n}^{+}=\left\{x=\sum_{0}^{\infty} x_{n} z^{n} \in H^{0}(D ; \dot{\mathfrak{g}}): x(0)=x_{0} \in \dot{\mathfrak{n}}^{+}\right\}
\end{aligned}
$$

and

$$
\mathfrak{n}^{-}=\left\{x=\sum_{0}^{\infty} x_{n} z^{-n} \in H^{0}\left(D^{*} ; \dot{\mathfrak{g}}\right): x(\infty)=x_{0} \in \dot{\mathfrak{n}}^{-}\right\} .
$$

This is compatible with the finite dimensional triangular decomposition (2.1). We let $N^{ \pm}$denote the profinite nilpotent groups corresponding to $\mathfrak{n}^{ \pm}$, e.g.

$$
N^{-}=H^{0}\left(D^{*}, \infty ; \dot{G}, \dot{N}^{-}\right) .
$$


There is a unique Ad-invariant symmetric bilinear form on $\widehat{L} \dot{\mathfrak{g}}$ which extends the normalized Killing form on $\dot{\mathfrak{g}}$. It has the following restriction to $\mathfrak{h}$ :

$$
\left\langle c_{1} d+c_{2} c+h, c_{1}^{\prime} d+c_{2}^{\prime} c+h^{\prime}\right\rangle=c_{1} c_{2}^{\prime}+c_{2} c_{1}^{\prime}+\left\langle h, h^{\prime}\right\rangle .
$$

This form is nondegenerate. The restriction of this form to $\widehat{L} \dot{u}$ is also nondegenerate, although this restriction is of Minkowski type, in contrast to the finite dimensional situation.

The simple roots for $(\widehat{L} \dot{\mathfrak{g}}, \mathfrak{h})$ are $\left\{\alpha_{j}: 0 \leq j \leq r k \dot{\mathfrak{g}}\right\}$, where

$$
\alpha_{0}=d^{*}-\theta, \quad \alpha_{j}=\dot{\alpha}_{j}, \quad j>0,
$$

$d^{*}(d)=1, d^{*}(c)=0, d^{*}(\dot{\mathfrak{h}})=0$, and the $\dot{\alpha}_{j}$ denote the simple roots for the triangular decomposition of $\dot{\mathfrak{g}}$ (with $\dot{\alpha}_{j}$ vanishing on $c$ and $d$ ). The simple coroots of $\mathfrak{h} \subset \widehat{L} \dot{\mathfrak{g}}$ are $\left\{h_{j}: 0 \leq j \leq r k \dot{\mathfrak{g}}\right\}$, where

$$
h_{0}=c-\dot{h}_{\theta}, \quad h_{j}=\dot{h}_{j}, \quad j>0,
$$

and the $\left\{\dot{h}_{j}\right\}$ are the simple coroots of $\dot{\mathfrak{g}}$. For $i>0$, the root homomorphism $i_{\alpha_{i}}$ is $i_{\dot{\alpha}_{i}}$ followed by the inclusion $\dot{\mathfrak{g}} \subset \widehat{L} \dot{\mathfrak{g}}$. For $i=0$

$$
i_{\alpha_{0}}\left(\left(\begin{array}{ll}
0 & 0 \\
1 & 0
\end{array}\right)\right)=e_{\theta} z^{-1}, \quad i_{\alpha_{0}}\left(\left(\begin{array}{ll}
0 & 1 \\
0 & 0
\end{array}\right)\right)=e_{-\theta} z
$$

where $\left\{e_{-\theta}, \dot{h}_{\theta}, e_{\theta}\right\}$ satisfy the $\operatorname{sl}(2, \mathbb{C})$-commutation relations, and $e_{\theta}$ is a highest root for $\dot{\mathfrak{g}}$.

Let $\Lambda_{j}$ denote the fundamental dominant integral functionals on $\mathfrak{h}$. Any linear function $\lambda$ on $\mathfrak{h}$ can be written uniquely as $\lambda=\dot{\lambda}+\lambda\left(h_{0}\right) \Lambda_{0}$, where $\dot{\lambda}$ can be identified with a linear function on $\dot{\mathfrak{h}}$. In particular $\delta$, the sum of the fundamental dominant integral functionals, is given by $\delta=\dot{\delta}+\dot{g} \Lambda_{0}$, where $\dot{\delta}$ is the sum of the fundamental dominant integral functionals for the finite dimensional triangular structure (2.1).

For $\tilde{g} \in N^{-} \cdot H \cdot N^{+} \subset \tilde{L} G$,

$$
\tilde{g}=l \cdot\left(\operatorname { d i a g } \tilde { ) } \cdot u , \quad \text { where } \quad \left(\operatorname{diag} \tilde{)}(\tilde{g})=\prod_{0}^{r k \dot{\mathfrak{g}}} \sigma_{j}(\tilde{g})^{h_{j}},\right.\right.
$$

where $\sigma_{j}=\sigma_{\Lambda_{j}}$ is the matrix coefficient corresponding to $\Lambda_{j}$. If $\tilde{g}$ projects to $g \in N^{-} \cdot \dot{H} \cdot N^{+} \subset$ $L G$, then because $\sigma_{0}^{h_{0}}=\sigma_{0}^{c-\dot{h}_{\theta}}$ projects to $\sigma_{0}^{-\dot{h}_{\theta}}$, we have $g=l \cdot \operatorname{diag} \cdot u$, where

$$
\operatorname{diag}(g)=\sigma_{0}(\tilde{g})^{-\dot{h}_{\theta}} \prod_{1}^{r k \dot{\mathfrak{g}}} \sigma_{j}(\tilde{g})^{\dot{h}_{j}}=\prod_{1}^{r k \dot{\mathfrak{g}}}\left(\frac{\sigma_{j}(\tilde{g})}{\sigma_{0}(\tilde{g})^{\check{a}_{j}}}\right)^{\dot{h}_{j}}
$$

and the $\check{a}_{j}$ are positive integers such that $\dot{h}_{\theta}=\sum \check{a}_{j} \dot{h}_{j}$.

If $\tilde{g} \in \tilde{L} K$, then $\left|\sigma_{j}(\tilde{g})\right|$ depends only on $g$, the projection of $\tilde{g}$ in $L K$. We will indicate this by writing

$$
\left|\sigma_{j}(\tilde{g})\right|=\left|\sigma_{j}\right|(g) .
$$

In this paper we will mainly deal with generic elements in $\tilde{L} K$ having diagonal elements with trivial $T$-component. Thus (2.6) has the practical consequence (important in Sections 6 and 7) that we can generally work with ordinary loops in $K$. We record this for later reference.

Lemma 1. The restriction of the projection $\tilde{L} K \rightarrow L K$ to generic elements with diagonal terms having trivial T-component is injective. 


\section{$3 \quad$ Type I case}

In this section we assume that $\dot{X}$ is a type I simply connected and irreducible symmetric space. We let $\dot{U}$ denote the universal covering of the identity component of the group of automorphisms of $\dot{X}$, and so on, as in the Introduction. The irreduciblity and type I conditions imply that $\dot{\mathfrak{u}}$ and $\dot{\mathfrak{g}}$ are simple Lie algebras.

Exactly as in the preceding section, we introduce the affine analogues $\mathfrak{g}=\widehat{L} \dot{\mathfrak{g}}$ and $\mathfrak{u}=\widehat{L} \dot{\mathfrak{u}}$ of $\dot{\mathfrak{g}}$ and its compact real form $\dot{\mathfrak{u}}$, respectively, and also the corresponding groups. We will write the corresponding Lie algebra involution as $-(\cdot)^{*}$, as we typically would in a finite dimensional matrix context.

Let $\Theta$ denote the involution corresponding to the pair $(\dot{\mathfrak{u}}, \dot{\mathfrak{k}})$. We extend $\Theta$ complex linearly to $\dot{\mathfrak{g}}$, and we use the same symbol to denote the involution for the Lie group $\dot{G}$. We assume that the triangular decomposition of the preceding section is $\Theta$-stable. We extend $\Theta$ to an involution of $L \dot{\mathfrak{g}}$ pointwise, and we then extend $\Theta$ to $\widehat{L} \dot{\mathfrak{g}}$ by

$$
\Theta(\mu d+x+\lambda c)=\mu d+\Theta(x)+\lambda c .
$$

The triangular decomposition for $\widehat{L} \dot{\mathfrak{g}}$ is $\Theta$-stable, and $\mathfrak{t}_{0}=\mathfrak{h} \cap \widehat{L} \dot{\mathfrak{k}}$ is maximal abelian in $\widehat{L} \dot{\mathfrak{k}}$. We let $\sigma$ denote the Lie algebra involution $-(\cdot)^{* \Theta}$, we use the same symbol for the corresponding group involution, and we let $\mathfrak{g}_{0}=\widehat{L} \dot{\mathfrak{g}}_{0}$ and $G_{0}=\widehat{L} \dot{G}_{0}$ denote the corresponding real forms.

We have defined the various objects in the diagram (1.1). The Lie algebra analogue of the diagram (1.1) is given by

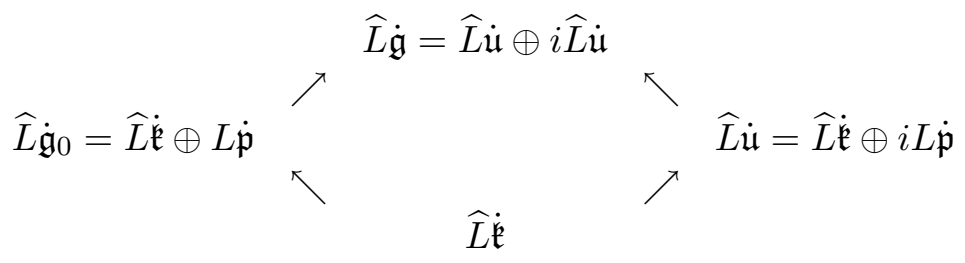

where $\widehat{L} \dot{\mathfrak{k}}=i \mathbb{R} d \propto \tilde{L} \dot{\mathfrak{k}}$ and $\tilde{L} \dot{\mathfrak{k}}=L \dot{\mathfrak{k}} \oplus i \mathbb{R} c$. The sums in the diagram represent Cartan decompositions. In analogy with [3], we will write $\mathfrak{p}=L \dot{\mathfrak{p}}, \mathfrak{h}_{0}=\mathfrak{h} \cap \mathfrak{g}_{0}=\mathfrak{t}_{0} \oplus \mathfrak{a}_{0}$ (relative to the Cartan decomposition for $\mathfrak{g}_{0}$ ), and $\mathfrak{t}=\mathfrak{h} \cap \mathfrak{u}=\mathfrak{t}_{0} \oplus i \mathfrak{a}_{0}$.

Our next task is to explain the diagram (1.2). There are isomorphisms induced by natural maps

$$
\widehat{L} \dot{U} / \widehat{L} \dot{K} \rightarrow \tilde{L} \dot{U} / \tilde{L} \dot{K} \rightarrow L \dot{U} / L \dot{K} \rightarrow L \dot{X}
$$

and

$$
\widehat{L} \dot{G}_{0} / \widehat{L} \dot{K} \rightarrow \tilde{L} \dot{G}_{0} / \tilde{L} \dot{K} \rightarrow L \dot{G}_{0} / L \dot{K} \rightarrow L \dot{X}_{0}
$$

In each case the first two maps are obviously isomorphisms. In the first and second cases the third map is an isomorphism because $\dot{X}$ and $\dot{X}_{0}$ are simply connected, respectively.

We will take full advantage of these isomorphisms, and consequently there will be times when we want to use the quotient involving hats, or tildes, and times when we want to use the quotient not involving hats, or tildes. To distinguish when we are using hats, we will write our group elements with hats, and similarly with tildes. Thus $\widehat{g}$ will typically denote an element of $\widehat{L} \dot{G}$, whereas $g$ will typically denote an element of $L \dot{G}$, and unless stated otherwise, these two elements will be related by projection.

For the natural maps

$$
\widehat{L} \dot{G} / \widehat{L} \dot{G}_{0} \rightarrow \tilde{L} \dot{G} / \tilde{L} \dot{G}_{0} \rightarrow L \dot{G} / L \dot{G}_{0} \rightarrow L\left(\dot{G} / \dot{G}_{0}\right)
$$


and

$$
\widehat{L} \dot{G} / \widehat{L} \dot{U} \rightarrow \tilde{L} \dot{G} / \tilde{L} \dot{U} \rightarrow L \dot{G} / L \dot{U} \rightarrow L(\dot{G} / \dot{U})
$$

in each case the third map is an isomorphism, but the first two maps fail to be isomorphisms. For example in (3.3) the second map is surjective, but there is a nontrivial fiber $\exp (\mathbb{R} c) \tilde{L} \dot{G}_{0}$ over the basepoint (represented by 1 ). This is the reason for the appearance of $\tilde{L} \dot{G} / \tilde{L} \dot{G}_{0}$, rather than $L\left(\dot{G} / \dot{G}_{0}\right)$, in the diagram (1.2).

There is an Iwasawa decomposition for $\widehat{L} \dot{G}$ (see Chapter 8 of [11]), which we write as

$$
\widehat{L} \dot{G} \simeq N^{-} \times A \times \widehat{L} \dot{U}: \widehat{g}=\mathbf{l}(\widehat{g}) \mathbf{a}(\widehat{g}) \mathbf{u}(\widehat{g}),
$$

where $A=\exp \left(\mathfrak{h}_{\mathbb{R}}\right)$. In analogy with [3], we also write $\mathbf{a}=\mathbf{a}_{0} \mathbf{a}_{1}$, relative to $\exp \left(\mathfrak{h}_{\mathbb{R}}\right)=$ $\exp \left(\mathfrak{a}_{0}\right) \exp \left(i \mathfrak{t}_{0}\right)$. There is an induced right action

$$
\widehat{L} \dot{U} \times\left(\widehat{T} \times \widehat{L} \dot{G}_{0}\right) \rightarrow \widehat{L} \dot{U}:\left(\widehat{u}, \widehat{t}, \widehat{g}_{0}\right) \rightarrow \widehat{t}^{-1} \mathbf{u}\left(\widehat{u} \widehat{g}_{0}\right)
$$

arising from the identification of $\widehat{L} \dot{U}$ with $N^{-} A \backslash \widehat{L} \dot{G}$. We also write $A_{0}=A \cap G_{0}$.

The Cartan embedding for the unitary type symmetric space is given by

$$
\phi: L(\dot{U} / \dot{K}) \rightarrow \tilde{L} \dot{U} \subset \widehat{L} \dot{U}: \tilde{u} \tilde{L} \dot{K} \rightarrow \tilde{u} \tilde{u}^{-\Theta},
$$

where we are using the isomorphism (3.1) in an essential way to express this mapping. There is a corresponding embedding $\psi$ in the dual case. More generally

$$
\phi: \tilde{L} \dot{G} / \tilde{L} \dot{G}_{0} \rightarrow \tilde{L} \dot{G} \subset \widehat{L} \dot{G}: \tilde{g} \tilde{L} \dot{G}_{0} \rightarrow \tilde{g} \tilde{g}^{* \Theta},
$$

and the extension of $\psi$ is similarly defined.

This explains the diagram (1.2). We should note that in what follows, in place of (1.1) and (1.2), and the Kac-Moody triangular decomposition (2.3) for $\mathfrak{g}$, we could simply consider the ordinary loop functor of the diagrams (0.1) and (0.2) of [3], and the analogue of the triangular decomposition for $L \dot{\mathfrak{g}}$. But in the process we would miss out on the interesting applications (such as Theorem 1), and in analyzing the resulting Hamiltonian systems we would inevitably be led to this Kac-Moody extended point of view.

We are now in a position to repeat verbatim the arguments in Sections 2-4 of [3], supplemented with remarks concerning Poisson structures in infinite dimensions. We will summarize the main points.

Proposition 1. Relative to the extended real form $\operatorname{Im}\langle\cdot, \cdot\rangle$ on $\mathfrak{g}=\widehat{L} \dot{\mathfrak{g}}$,

$$
\left(\mathfrak{g}, \mathfrak{u}, \mathfrak{h}_{\mathbb{R}} \oplus \mathfrak{n}^{-}\right) \quad \text { and } \quad\left(\mathfrak{g}, \mathfrak{g}_{0}, \mathfrak{t} \oplus \mathfrak{n}^{-}\right)
$$

are Manin triples, extending the finite dimensional Manin triples $\left(\dot{\mathfrak{g}}, \dot{\mathfrak{u}}, \dot{\mathfrak{h}}_{\mathbb{R}} \oplus \dot{\mathfrak{n}}^{-}\right)$and $\left(\dot{\mathfrak{g}}, \dot{\mathfrak{g}}_{0}, \dot{\mathfrak{t}} \oplus \dot{\mathfrak{n}}^{-}\right)$, respectively.

We next apply the Evens-Lu construction to obtain global Poisson structures $\Pi_{X}$ and $\Pi_{X_{0}}$ on the loop spaces $X=L \dot{X}$ and $X_{0}=L \dot{X}_{0}$, respectively, using the isomorphisms (3.1) and (3.2). These Poisson structures are given by the same formulas as in the finite dimensional cases: see (3.1) and (4.1) of [3]. As in the finite dimensional case, we have used the Ad-invariant symmetric form on $\widehat{L} \dot{\mathfrak{g}}$ to identify $\mathfrak{p}$ with a subspace of its dual (note the form is definite on $\mathfrak{p}$ ). However, in this infinite dimensional context, the inclusion $\mathfrak{p} \rightarrow \mathfrak{p}^{*}$ is proper, so that this Poisson structure must be understood in a weak sense. Consequently it is not clear that we can appeal to any general theory (e.g. as in [8]) for the existence of a symplectic foliation, etc. 
As in [3], the Hilbert transform $\mathcal{H}: \mathfrak{g} \rightarrow \mathfrak{g}$ associated to the triangular decomposition of $\mathfrak{g}$ is given by

$$
x=x_{-}+x_{0}+x_{+} \mapsto \mathcal{H}(x)=-i x_{-}+i x_{+} .
$$

In the following statement, we can, and do, view $\mathbf{a}_{0}$ (defined following (3.4)) as a function on $X=G_{0} / K$.

\section{Theorem 3.}

(a) The Poisson structure $\Pi_{X_{0}}$ has a regular symplectic foliation (by weak symplectic manifolds), given by the level sets of the function $\mathbf{a}_{0}$.

(b) The horizontal parameterization for the symplectic leaf through the basepoint is given by the map $s: A_{0} \backslash G_{0} / K \rightarrow G_{0} / K$

$$
A_{0} g_{0} K \rightarrow s\left(A_{0} g_{0} K\right)=\mathbf{a}_{0}^{-1} g_{0} K,
$$

where $g_{0}=\mathbf{l a}_{0} \mathbf{a}_{1} \mathbf{u}$.

(c) If we identify $T\left(G_{0} / K\right)$ with $G_{0} \times_{K} \mathfrak{p}$ in the usual way, then

$$
\omega_{1}\left(\left[g_{0}, x\right] \wedge\left[g_{0}, y\right]\right)=\left\langle\operatorname{Ad}\left(\mathbf{u}\left(g_{0}\right)^{-1}\right) \circ \mathcal{H} \circ \operatorname{Ad}\left(\mathbf{u}\left(g_{0}\right)\right)(x), y\right\rangle
$$

is a well-defined two-form on $G_{0} / K$.

(d) Along the symplectic leaves, $\Pi_{X_{0}}^{-1}$ agrees with the restriction of the closed two-form $\omega_{1}$.

Note that the facts that the form $\omega_{1}$ is closed and nondegenerate (on the double coset space $\left.A_{0} \backslash G_{0} / K \rightarrow G_{0} / K\right)$ is proven directly in Section 1 of [3].

\section{Theorem 4.}

(a) The Poisson structure $\Pi_{X}$ has a symplectic foliation (by weak symplectic manifolds). The symplectic leaves are identical to the projections of the $L \dot{G}_{0}$-orbits, for $L \dot{G}_{0}$ acting on $L \dot{U}$ as in $(3.5)$, to $L(\dot{U} / \dot{K})$. Let $S(1)$ denote the symplectic leaf containing the identity.

(b) The action of $\widehat{T}_{0}=\operatorname{Rot}\left(S^{1}\right) \times T_{0} \times \exp (i \mathbb{R} c)$ on $\Sigma_{1}^{\phi(L(\dot{U} / \dot{K}))}$ is Hamiltonian with momentum mapping

$$
\Sigma_{1}^{\phi(L(\dot{U} / \dot{K}))} \rightarrow\left(\widehat{\mathfrak{t}}_{0}\right)^{*}: \tilde{u} \rightarrow\left\langle-\frac{i}{2} \log \left(a_{\phi}(\tilde{u}), \cdot\right\rangle,\right.
$$

where $\tilde{u}$ has the unique triangular decomposition $\tilde{u}=\operatorname{lm} \tilde{a}_{\phi}{ }^{* \Theta}$.

(c) The map $\tilde{\mathbf{u}}: G_{0} \rightarrow U$

$$
g_{0} \mapsto \mathbf{u}\left(g_{0}\right),
$$

where $\mathbf{u}$ is defined by (3.4), is equivariant for the right actions of $K$ on $G_{0}$ and $U$, invariant under the left action of $A_{0}$ on $G_{0}$ and descends to a $T_{0}$-equivariant diffeomorphism

$$
\tilde{\mathbf{u}}: A_{0} \backslash G_{0} / K \rightarrow S(1) .
$$

This induces an isomorphism of $T_{0}$-Hamiltonian spaces

$$
\left(A_{0} \backslash G_{0} / K, \omega_{1}\right) \rightarrow\left(S(1), \Pi_{X}^{-1}\right),
$$

where $\omega_{1}$ is as in (3.6).

The symplectic foliation in part (a) can be described in a completely explicit way in terms of triangular factorization and the Cartan embedding $\phi$ (see [2] for the finite dimensional case; the arguments there extend directly).

Throughout this paper we will focus on the generic system $S(1)$ in part (c). As we mentioned in the Introduction, the main application which we envision is to use this Hamiltonian system to generate useful integral formulas. In this loop context these integrals are infinite dimensional, and more infrastructure and analysis are required to properly formulate and justify them (see [9], especially Section 7). Even in finite dimensions, it is not known whether these type I systems have any integrability properties (in sharp contrast to the type II case). 


\section{The $S^{2}$ case}

In this section we will do some illustrative calculations in the simplest Type I case

$$
G_{0}=\hat{L} S U(1,1) \nearrow \begin{gathered}
G=\hat{L} S L(2, \mathbb{C}) \\
K=\hat{L} U(1)
\end{gathered} \nearrow U=\hat{L} S U(2)
$$

If we identify $\dot{X}_{0}$ with $\Delta$ (the unit disk) and $\dot{X}$ with $\hat{\mathbb{C}}$ in the usual way, then from the preceding section we have maps

$$
L \Delta \stackrel{\tilde{\mathbf{u}}}{\rightarrow} L \hat{\mathbb{C}} \stackrel{\phi}{\rightarrow} \tilde{L} S U(2),
$$

where the map $\tilde{\mathbf{u}}$ is covered by the map

$$
\hat{L} S U(1,1) \stackrel{\mathbf{u}}{\rightarrow} \hat{L} S U(2)
$$

induced by the Iwasawa decomposition $g_{0}=\mathbf{l}\left(g_{0}\right) \mathbf{a}\left(g_{0}\right) \mathbf{u}\left(g_{0}\right)$.

To orient the reader, we recall the nonloop case:

$$
\begin{aligned}
& S U(1,1) \stackrel{\mathbf{u}}{\rightarrow} S U(2) \\
& \downarrow \quad \downarrow \\
& \Delta \quad \rightarrow \quad \hat{\mathbb{C}} \quad \stackrel{\phi}{\rightarrow} S U(2) \\
& g_{0}=\frac{1}{(1-Z \bar{Z})^{1 / 2}}\left(\begin{array}{cc}
1 & \bar{Z} \\
Z & 1
\end{array}\right) \rightarrow \mathbf{u}\left(g_{0}\right)=\frac{1}{(1+Z \bar{Z})^{1 / 2}}\left(\begin{array}{cc}
1 & \bar{Z} \\
-Z & 1
\end{array}\right) \\
& Z \quad \rightarrow \quad-Z \quad \rightarrow \frac{1}{1+|Z|^{2}}\left(\begin{array}{cc}
1-|Z|^{2} & 2 \bar{Z} \\
-2 Z & 1-|Z|^{2}
\end{array}\right)
\end{aligned}
$$

In this context $\mathbf{u}$ is obtained by a Gram-Schmidt process from the rows of $g_{0}$, and

$$
\mathbf{a}=\left(\frac{1+Z \bar{Z}}{1-Z \bar{Z}}\right)^{\frac{1}{2} h_{1}} .
$$

To calculate the symplectic form note that

$$
\begin{aligned}
{\left[g_{0}, X\right.} & \left.=\left(\begin{array}{ll}
0 & \bar{x} \\
x & 0
\end{array}\right)\right]\left.\rightarrow \frac{d}{d t}\right|_{t=0} Z\left(g_{0} e^{t X}\right) \\
& =\left.\frac{d}{d t}\right|_{t=0}(Z \operatorname{ch}(t x)+\operatorname{sh}(t x))(\operatorname{ch}(t x)+\bar{Z} \operatorname{sh}(t x))^{-1}=(1-Z \bar{Z}) x .
\end{aligned}
$$

Thus a variation $\dot{Z}$ of $Z$ will correspond to $\left[g_{0}, X\right]$ with $x=(1-Z \bar{Z})^{-1} \dot{Z}$. Thus

$$
\begin{aligned}
& \omega\left(\left[g_{0}, X\right] \wedge\left[g_{0}, Y\right]\right) \\
&=\omega\left(\left[\frac{1}{(1-Z \bar{Z})^{1 / 2}}\left(\begin{array}{cc}
1 & \bar{Z} \\
Z & 1
\end{array}\right),\left(\begin{array}{cc}
0 & \bar{x} \\
x & 0
\end{array}\right)\right] \wedge\left[\frac{1}{(1-Z \bar{Z})^{1 / 2}}\left(\begin{array}{cc}
1 & \bar{Z} \\
Z & 1
\end{array}\right),\left(\begin{array}{ll}
0 & \bar{y} \\
y & 0
\end{array}\right)\right]\right) \\
&=\left\langle\mathcal { H } \left(\operatorname{Ad}\left(\frac{1}{(1+Z \bar{Z})^{1 / 2}}\left(\begin{array}{cc}
1 & \bar{Z} \\
-Z & 1
\end{array}\right)\right)\left(\left(\begin{array}{cc}
0 & \bar{x} \\
x & 0
\end{array}\right)\right)\right.\right. \\
&\left.\wedge \operatorname{Ad}\left(\frac{1}{(1+Z \bar{Z})^{1 / 2}}\left(\begin{array}{cc}
1 & \bar{Z} \\
-Z & 1
\end{array}\right)\right)\left(\left(\begin{array}{cc}
0 & \bar{y} \\
y & 0
\end{array}\right)\right)\right\rangle=\frac{i}{\left(1-|Z|^{4}\right)}\left(\bar{Z} Z^{\prime}-\dot{Z} \bar{Z}^{\prime}\right) .
\end{aligned}
$$


Thus

$$
\omega=\frac{i}{\left(1-|Z|^{4}\right)} d Z \wedge d \bar{Z} .
$$

Returning to the loop case, we denote the maps in (4.1) by

$$
f(\theta) \rightarrow F(\theta) \rightarrow \phi(F)(\theta) .
$$

We have written the argument as $\theta$, as a reminder that these are functions on $S^{1}$. To calculate the map $f \rightarrow F$, we need to find the Iwasawa decomposition

$$
g_{0}(\theta)=\frac{1}{(1-f(\theta) \bar{f}(\theta))^{1 / 2}}\left(\begin{array}{cc}
1 & \bar{f}(\theta) \\
f(\theta) & 1
\end{array}\right)=\mathbf{l}(z) \mathbf{a u}(\theta)
$$

and remember that $\mathbf{l}(z)$ extends to a holomorphic function in the exterior of $S^{1}$. In turn

$$
\phi(F)(\theta)=\mathbf{u u}^{* \Theta}=l(z) a u(z),
$$

where $l=\mathbf{a}^{-1} \mathbf{l}^{-1} \mathbf{a}, a=\mathbf{a}^{-2}=\left|\sigma_{0}\right|^{h_{0}}\left|\sigma_{1}\right|^{h_{1}}, u=l^{* \Theta}$. The image of $\phi(F)$ in $L S U(2)$ has the form

$$
\left(\begin{array}{cc}
\alpha(\theta) & \beta(\theta) \\
-\bar{\beta}(\theta) & \bar{\alpha}(\theta)
\end{array}\right)=l\left(z^{-1}\right)\left(\frac{\left|\sigma_{1}\right|}{\left|\sigma_{0}\right|}\right)^{h_{1}} u(z)
$$

(see $(2.5))$.

The Iwasawa decomposition of $g_{0}$ (the special self-adjoint representative above) is equivalent to

$$
g_{0}^{*} \mathbf{l}\left(g_{0}\right)^{-*} \mathbf{a}\left(g_{0}\right)^{-1}=g_{0} \mathbf{l}\left(g_{0}\right)^{-*} \mathbf{a}\left(g_{0}\right)^{-1}=\mathbf{u}\left(g_{0}\right) .
$$

Write $\mathbf{1}^{-*}=\left(\begin{array}{ll}a & b \\ c & d\end{array}\right)$, so that $a, b, c, d$ are holomorphic functions in $D, a(0)=d(0)=1$, and $c(0)=0$. Then

$$
\left(\begin{array}{ll}
1 & f \\
f & 1
\end{array}\right)\left(\begin{array}{ll}
a & b \\
c & d
\end{array}\right)\left(\begin{array}{cc}
a_{0}^{-1} & 0 \\
0 & a_{0}
\end{array}\right)
$$

is of the form $\left(\begin{array}{cc}A & B \\ -\bar{B} & \bar{A}\end{array}\right)$. This implies

$$
a+\bar{f} c=(\overline{f b}+\bar{d}) a_{0}^{2}, \quad f a+c=-(\bar{b}+f \bar{d}) a_{0}^{2} .
$$

As a reminder, these are equations for functions defined on $S^{1}$.

Let $\mathcal{H}_{0}=P_{+}-P_{-}$, where for a scalar function $g=\sum g_{n} z^{n}, P_{+} g=\sum_{n \geq 0} g_{n} z^{n}$. We take the conjugate of the first equation in (4.2) and rewrite it as

$$
-\mathcal{H}_{0}\left(\bar{a}+d a_{0}^{2}\right)+2=f \mathcal{H}_{0}\left(b a_{0}^{2}+\bar{c}\right) .
$$

This is equivalent to

$$
-\left(\bar{a}+d a_{0}^{2}\right)+2=\mathcal{H}_{0} f \mathcal{H}_{0}\left(b a_{0}^{2}+\bar{c}\right) .
$$

The second equation in (4.2) is equivalent to

$$
\bar{f}\left(\bar{a}+d a_{0}^{2}\right)=-\left(b a_{0}^{2}+\bar{c}\right) .
$$


These two equations imply

$$
\left(b a_{0}^{2}+\bar{c}\right)=-2\left(1-\bar{f} \mathcal{H}_{0} f \mathcal{H}_{0}\right)^{-1}(\bar{f})=-2 \bar{f}\left(1-\mathcal{H}_{0} f \mathcal{H}_{0} \bar{f}\right)^{-1}(1)
$$

Note that the inverse on the right exists, because $\sup \left\{|f(z)|: z \in S^{1}\right\}<1$. This determines $b a_{0}^{2}$ and $c$, by applying $P_{ \pm}$.

We now see that

$$
\bar{a}+d a_{0}^{2}=2\left(1+\mathcal{H}_{0} f \mathcal{H}_{0} \bar{f}\left(1-\mathcal{H}_{0} f \mathcal{H}_{0} \bar{f}\right)^{-1}(1)\right)=2\left(1-\mathcal{H}_{0} f \mathcal{H}_{0} \bar{f}\right)^{-1}(1) .
$$

Note that $1+a_{0}^{2}$ is the zero mode of the right hand side, so that in principle we have determined $a_{0}$, and $\mathbf{l}$. This form of the solution does not explain in a clear way why the zero mode of the right hand side is $>2$.

To summarize, let

$$
h=2\left(1-\mathcal{H}_{0} f \mathcal{H}_{0} \bar{f}\right)^{-1}(1)
$$

(this is a well-defined function on $S^{1}$, and we do not know much more about it). Then

$$
\bar{c}=-P_{-}(\bar{f} h), \quad b a_{0}^{2}=-P_{+}(\bar{f} h), \quad \bar{a}-1=P_{-}(h), \quad 1+d a_{0}^{2}=P_{+}(h) .
$$

This implies the following

\section{Proposition 2.}

$$
\mathbf{u}=g_{0} \mathbf{l}^{-*} \mathbf{a}^{-1}=(1-f \bar{f})^{-1 / 2} a_{0}^{-1}\left(\begin{array}{cc}
\left(1+P_{-} h-f P_{-}(\bar{f} h)\right)^{*} & -\left(\bar{f}+\bar{f} P_{-} h-P_{-}(\bar{f} h)\right) \\
\left(\bar{f}+\bar{f} P_{-} h-P_{-}(\bar{f} h)\right)^{*} & 1+P_{-} h-f P_{-}(\bar{f} h)
\end{array}\right)
$$

and

$$
F=\left(\frac{\bar{f}+\bar{f} P_{-} h-P_{-}(\bar{f} h)}{1+P_{-} h-f P_{-}(\bar{f} h)}\right)^{*} \in L \hat{\mathbb{C}} .
$$

These general formulas are not especially enlightening. However, the $S U(2)$ case considered below suggests that there might be some special cases of these formulas which are tractable.

\section{Type II case}

In the type II case there is more than one reasonable interpretation of the diagram (1.1). The differences between the possibilities are minor, but potentially confusing. We will briefly describe a first possibility, which leads to diagram (1.2), but we will then consider a second possibility, which is more elementary in a technical sense, and we will pursue this in detail.

Throughout this section $\dot{K}$ denotes a simply connected compact Lie group with simple Lie algebra $\dot{\mathfrak{k}}, \dot{X}=\dot{K}$, viewed as a symmetric space, $\dot{U}=\dot{K} \times \dot{K}$, and $\dot{\mathfrak{g}}=\dot{\mathfrak{k}} \mathbb{C} \oplus \dot{\mathfrak{k}}^{\mathbb{C}}$.

In the first interpretation of diagram (1.1), $\mathfrak{g}=\hat{L} \dot{\mathfrak{g}}$ is defined in the following way. We first define a central extension

$$
0 \rightarrow \mathbb{C} c \rightarrow \tilde{L} \dot{\mathfrak{g}} \rightarrow L \dot{\mathfrak{g}} \rightarrow 0
$$

As a vector space

$$
\tilde{L} \dot{\mathfrak{g}}=L \dot{\mathfrak{g}} \oplus \mathbb{C} c
$$


the bracket is defined as in $(2.2)$, where the form $\langle\cdot, \cdot\rangle$ is the sum of the normalized invariant symmetric forms for the two $\dot{\mathfrak{k}}^{\mathbb{C}}$ factors:

$$
\langle(x, y),(X, Y)\rangle=\langle x, y\rangle+\langle X, Y\rangle \text {. }
$$

Then $\hat{L} \dot{\mathfrak{g}}=\mathbb{C} d \propto \tilde{L} \dot{\mathfrak{g}}$, and

$$
\Theta(\lambda d+(x, y)+\mu c)=\lambda d+(y, x)+\mu c .
$$

The Lie algebra analogue of diagram (1.1) is

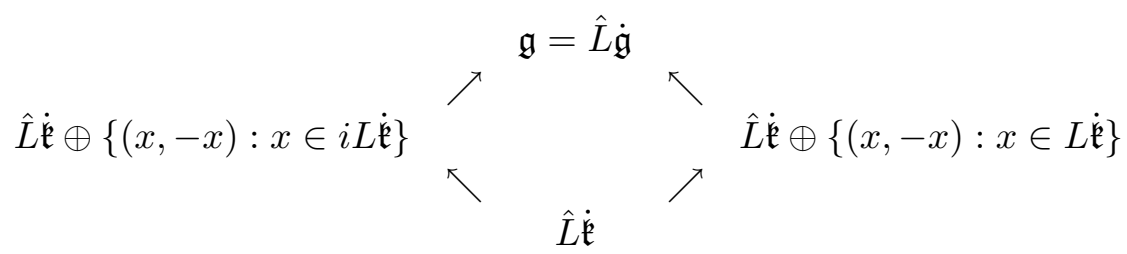

where $\hat{L} \dot{\mathfrak{k}}=i \mathbb{R} d \propto \tilde{L} \dot{\mathfrak{k}}$ and $\tilde{L} \dot{\mathfrak{k}}=\{(x, x): x \in L \dot{\mathfrak{k}}\} \oplus i \mathbb{R} c$.

At the group level $G=\hat{L} \dot{G}=\mathbb{C} \propto \tilde{L} \dot{G}$ where $\tilde{L} \dot{G}$ is an extension of $L \dot{G}$ by $\mathbb{C}^{*}$; precisely, $\tilde{L} \dot{G}$ is a quotient

$$
0 \rightarrow \mathbb{C}^{*} \rightarrow \tilde{L} \dot{K}^{\mathbb{C}} \times \tilde{L} \dot{K}^{\mathbb{C}} \rightarrow \tilde{L} \dot{G} \rightarrow 0
$$

where $\lambda \in \mathbb{C}^{*}$ maps antidiagonally, $\lambda \rightarrow\left(\lambda^{c}, \lambda^{-c}\right)$.

As in the type I case, there are isomorphisms

$$
\hat{L} \dot{U} / \hat{L} \dot{K} \rightarrow \tilde{L} \dot{U} / \tilde{L} \dot{K} \rightarrow L \dot{U} / L \dot{K} \rightarrow L \dot{K}
$$

where the last map is given by $\left(k_{1}, k_{2}\right) \rightarrow k_{1} k_{2}^{-1}$. The Cartan embedding is given by

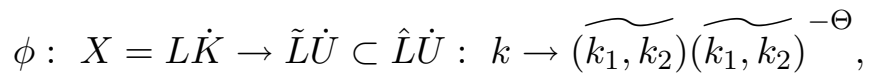

where $k=k_{1} k_{2}^{-1}$. The dual map $\psi$ is described in a similar way. This leads to the diagram (1.2) in this type II case.

This first interpretation of diagram (1.1) is somewhat inconvenient, because unlike the finite dimensional case, $X \neq K$, and $U \neq K \times K$. In the remainder of this paper we will consider a setup where these equalities do hold. It will be easier to compare this setup with the finite dimensional case. The modest price we pay is that, in this second interpretation, $X$ is a covering of $L \dot{K}$ (also, as a symmetric space, the invariant geometric structure is of Minkowski type, rather than Riemannian type, but this geometric structure is irrelevant for our purposes).

From now on, we set $K=\widehat{L} \dot{K}$, as in Section 2. We henceforth understand the diagram (1.1) to be

$$
G_{0}=\left\{g_{0}=\left(g, g^{-*}\right): g \in K^{\mathbb{C}}\right\} \nearrow_{\nwarrow} \quad \begin{gathered}
G=K^{\mathbb{C}} \times K^{\mathbb{C}} \\
\Delta(K)=\{(k, k): k \in K\}
\end{gathered} \nearrow^{\nearrow} U=K \times K
$$

where $\Delta(K)=\{(k, k): k \in K\}, G_{0}=\left\{g_{0}=\left(g, g^{-*}\right): g \in K^{\mathbb{C}}\right\}, K=\widehat{L} \dot{K}, G=\widehat{L} \dot{G}$, and the involution $\Theta$ is the outer automorphism $\Theta\left(\left(g_{1}, g_{2}\right)\right)=\left(g_{2}, g_{1}\right)$. Also

$$
X_{0}=G_{0} / \Delta(K) \simeq K^{\mathbb{C}} / K, \text { and } X=U / \Delta(K) \simeq K,
$$


where the latter isometry is $\left(k_{1}, k_{2}\right) \Delta(K) \mapsto k=k_{1} k_{2}^{-1}$. As in [3] we will use superchecks to distinguish structures for $k^{\mathbb{C}}$ versus those for $\mathfrak{g}$.

We fix a triangular decomposition

$$
\check{\mathfrak{g}}=\mathfrak{k}^{\mathbb{C}}=\check{\mathfrak{n}}_{-}+\check{\mathfrak{h}}+\check{\mathfrak{n}}_{+} .
$$

This induces a $\Theta$-stable triangular decomposition for $\mathfrak{g}$

$$
\mathfrak{g}=\underbrace{\left(\check{\mathfrak{n}}^{-} \times \check{\mathfrak{n}}^{-}\right)}_{\mathfrak{n}^{-}}+\underbrace{(\check{\mathfrak{h}} \times \check{\mathfrak{h}})}_{\mathfrak{h}}+\underbrace{\left(\check{\mathfrak{h}}^{+} \times \check{\mathfrak{n}}^{+}\right)}_{\mathfrak{n}^{+}} .
$$

Let $\check{\mathfrak{a}}=\check{\mathfrak{h}}_{\mathbb{R}}$ and $\check{\mathfrak{t}}=i \check{\mathfrak{a}}$. Then

$$
\mathfrak{t}_{0}=\{(x, x): x \in \mathfrak{\mathfrak { t }}\}, \quad \text { and } \quad \mathfrak{a}_{0}=\{(y,-y): y \in \check{\mathfrak{a}}\} .
$$

The standard Poisson Lie group structure on $U=K \times K$ induced by the decomposition in (5.2) is then the product Poisson Lie group structure for the standard Poisson Lie group structure on $K$ induced by the decomposition (5.1).

Let us denote the Poisson Lie group structure on $K$ by $\pi_{K}$ and the Evens-Lu homogeneous Poisson structure on $X=K$ by $\Pi_{X}$. The formal identification of $\mathfrak{k}$ with its dual via the invariant form allows us to view the Hilbert transform $\check{\mathcal{H}}$ associated to (5.1) as an element of $\mathfrak{k} \wedge \mathfrak{k}$. As a bivector field

$$
\pi_{K}=\check{\mathcal{H}}^{r}-\check{\mathcal{H}}^{l}
$$

where $\check{\mathcal{H}}^{r}$ (resp. $\check{\mathcal{H}}^{l}$ ) denotes the right (resp. left) invariant bivector field on $K$ generated by $\check{H}$, whereas $\Pi_{K}=\check{\mathcal{H}}^{r}+\check{\mathcal{H}}^{l}$.

Just as in the Type I case, the arguments of Sections 2-4 of [3] apply verbatim. We will focus on the new issues which arise.

As we pointed out in the Introduction, the first thing to note is that Theorem 5.1 of [3] does not hold in this context. The symplectic leaves for the Poisson Lie group structure on $K$ are finite dimensional, whereas the symplectic leaves for the Evens-Lu Poisson structure are finite codimensional. Thus these structures are fundamentally different.

As in [3], we will write

$$
k(\zeta)=\left(\begin{array}{ll}
1 & 0 \\
\zeta & 1
\end{array}\right)\left(\begin{array}{cc}
a(\zeta) & 0 \\
0 & a(\zeta)^{-1}
\end{array}\right)\left(\begin{array}{cc}
1 & -\bar{\zeta} \\
0 & 1
\end{array}\right)
$$

where $a(\zeta)=\left(1+|\zeta|^{2}\right)^{-1 / 2}$. Given a simple positive root $\gamma, i_{\gamma}: S U(2) \mapsto K$ denotes the root subgroup inclusion (as in (2.4)), and

$$
r_{\gamma}=i_{\gamma}\left(\left(\begin{array}{ll}
0 & i \\
i & 0
\end{array}\right)\right)
$$

a fixed representative for the corresponding Weyl group reflection.

Conjecture 1. Fix $w \in W$.

(a) The submanifold $\check{N}^{-} \cap w^{-1} \check{N}^{+} w \subset \check{N}^{-}$is $\check{T}$-invariant and symplectic.

Fix a representative $\mathbf{w}$ for $w$ with minimal factorization $\mathbf{w}=r_{n} \cdots r_{1}$, in terms of simple reflections $r_{j}=r_{\gamma_{j}}$ corresponding to simple positive roots $\gamma_{j}$. Let $\mathbf{w}_{j}=r_{j} \cdots r_{1}$.

(b) The map

$$
\mathbb{C}^{n} \rightarrow N^{-} \cap w^{-1} N^{+} w: \zeta=\left(\zeta_{n}, \ldots, \zeta_{1}\right) \rightarrow l(\zeta),
$$


where

$$
\mathbf{w}_{n-1}^{-1} i_{\gamma_{n}}\left(k\left(\zeta_{n}\right)\right) \mathbf{w}_{n-1} \cdots \mathbf{w}_{1}^{-1} i_{\gamma_{2}}\left(k\left(\zeta_{2}\right)\right) \mathbf{w}_{1} i_{\gamma_{1}}\left(k\left(\zeta_{1}\right)\right)=l(\zeta) a u
$$

is a diffeomorphism.

(c) In these coordinates the restriction of $\omega$ is given by

$$
\left.\omega\right|_{N^{-} \cap w^{-1} N^{+} w}=\sum_{j=1}^{n} \frac{i}{\left\langle\gamma_{j}, \gamma_{j}\right\rangle} \frac{1}{\left(1+\left|\zeta_{j}\right|^{2}\right)} d \zeta_{j} \wedge d \bar{\zeta}_{j},
$$

the momentum map is the restriction of $-\left\langle\frac{i}{2} \log (a), \cdot\right\rangle$, where

$$
a(k(\zeta))=\prod_{j=1}^{n}\left(1+\left|\zeta_{j}\right|^{2}\right)^{-\frac{1}{2} w_{j-1}^{-1} h_{\gamma_{j}} w_{j-1}},
$$

and Haar measure (unique up to a constant) is given by

$$
\begin{aligned}
d \lambda_{N^{-} \cap w^{-1} N^{+} w}(l) & =\prod_{j=1}^{n}\left(1+\left|\zeta_{j}\right|^{2}\right)^{\check{\delta}\left(w_{j-1}^{-1} h_{\gamma_{j}} w_{j-1}\right)-1} d \lambda\left(\zeta_{j}\right) \\
& =\prod_{1 \leq i<j \leq n}\left(1+\left|\zeta_{j}\right|^{2}\right)^{-\gamma_{i}\left(w_{i-1} w_{j-1}^{-1} h_{\gamma_{j}} w_{j-1} w_{i-1}^{-1}\right)} d \lambda\left(\zeta_{j}\right) .
\end{aligned}
$$

where $\check{\delta}=\sum \check{\Lambda}_{j}$, the sum of the dominant integral functionals for $\check{g}$, relative to (5.1).

(d) Let $C_{\mathbf{w}}$ denote the symplectic leaf through $\mathbf{w}$, with respect to $\pi_{K}$, with the negative of the induced symplectic structure. Then left translation by $\mathbf{w}^{-1}$ induces a symplectomorphism from $C_{\mathbf{w}}$, with its image in $(S(1), \omega)$, which is identified with $\check{N}^{-} \cap w^{-1} \check{N}^{+} w \subset \check{N}^{-}$.

Proposition 3. The following are true:

(1) part (b) of the conjecture;

(2) the formulas for $a(k(\zeta))$ and Haar measure in part (c);

(3) the right hand side of (5.4) equals the image of the symplectic structure for $C_{\mathbf{w}}$ with respect to the map in part (d); and

(4) the momentum maps for $\omega$ and the form in (3) do agree, and are given by the formula in $(\mathrm{c})$.

Thus the basic open question is whether (d) holds. This is known to be true in the finite dimensional case (see Theorems 5.1 and 5.2 of [3]).

Proof. The proof uses a number of facts which are recalled in Appendix A. We will freely use the notation which is used there. One technical point which emerges is that we are currently using $K$ to denote an extension of the real analytic completion of $L \dot{K}$ (and similarly for its Lie algebra, etc), whereas in the Appendix $K$ is the restriction of the extension to the polynomial loop group $L_{\mathrm{pol}} \dot{K}$. Since all the root homomorphisms map into the extension over the polynomial loop group, we will simply replace $K$ by this small subgroup, rather than introducing more notation.

Via the projection,

$$
K \rightarrow K / \check{T}=\check{G} / \check{B}_{+},
$$

$S(1)$ is identified with $\Sigma_{1}$, the unique open Birkhoff stratum in the flag manifold. There is a surjective map

$$
S L(2, \mathbb{C}) \times \cdots \times S L(2, \mathbb{C}) \rightarrow w^{-1} \bar{C}_{w}:
$$




$$
\left(g_{n}, \ldots, g_{1}\right) \rightarrow \mathbf{w}_{n-1}^{-1} i_{\gamma_{n}}\left(g_{n}\right) \mathbf{w}_{n-1} \cdots \mathbf{w}_{1}^{-1} i_{\gamma_{2}}\left(g_{2}\right) \mathbf{w}_{1} i_{\gamma_{1}}\left(g_{1}\right) \check{B}_{+} .
$$

This map is obtained by composing the Bott-Samelson desingularization of the Schubert variety $\bar{C}_{w}$ by left translation by $\mathbf{w}^{-1}$, as in Corollary 3 . This map has the remarkable property that the notion of generic is compatible with factorization: the preimage of $\mathbf{w}^{-1} C_{w}$ is precisely

$$
S L(2, \mathbb{C})^{\prime} \times \cdots \times S L(2, \mathbb{C})^{\prime},
$$

where

$$
S L(2, \mathbb{C})^{\prime}=\left\{\left(\begin{array}{ll}
a & b \\
c & d
\end{array}\right): a \neq 0\right\} .
$$

This follows from Corollary 3.

In terms of the affine coordinate $\check{N}^{-}$for $\check{G} / \check{B}^{+}$,

$$
\check{N}^{-} \cap w^{-1} \check{N}^{+} w \leftrightarrow w^{-1} C_{w} .
$$

We thus obtain a surjective map

$$
S L(2, \mathbb{C})^{\prime} \times \cdots \times S L(2, \mathbb{C})^{\prime} \rightarrow \check{N}^{-} \cap w^{-1} \check{N}^{+} w:\left(g_{n}, \ldots, g_{1}\right) \rightarrow l,
$$

where

$$
\mathbf{w}_{n-1}^{-1} i_{\gamma_{n}}\left(g_{n}\right) \mathbf{w}_{n-1} \cdots \mathbf{w}_{1}^{-1} i_{\gamma_{2}}\left(g_{2}\right) \mathbf{w}_{1} i_{\gamma_{1}}\left(g_{1}\right) \check{B}^{+}=l \check{B}^{+} .
$$

By part (a) of Corollary 4, this map induces a parameterization

$$
\begin{aligned}
& \mathbb{C}^{n} \leftrightarrow S(1)^{S U(2)} \times \cdots \times S(1)^{S U(2)} \rightarrow \check{N}^{-} \cap w^{-1} \check{N}^{+} w, \\
& \left(\zeta_{n}, \ldots, \zeta_{1}\right) \leftrightarrow\left(k_{n}\left(\zeta_{n}\right), \ldots, k_{1}\left(\zeta_{1}\right)\right) \rightarrow l(\zeta),
\end{aligned}
$$

where

$$
\mathbf{w}_{n-1}^{-1} i_{\gamma_{n}}\left(k_{n}\right) \mathbf{w}_{n-1} \cdots i_{\gamma_{1}}\left(k_{1}\right)=l(\zeta) \prod_{1}^{n} a\left(\zeta_{j}\right)^{\operatorname{Ad}\left(w_{j-1}^{-1}\right)\left(h_{\gamma_{j}}\right)} u,
$$

$u \in \check{N}^{+}$. This is part (b) of the Theorem.

The formula for $a(k(\zeta))$ in part (c) follows from Proposition 5. The formula for the Haar measure follows from part (b) of Corollary 4.

Finally part (3) of the Proposition is equivalent to Lu's factorization result, Theorem 3.4 of [7], and part (4) follows from the results in Sections 2-4 of [3] (which, as we have already noted, are valid in the loop context), and Theorem 3.4 of [7].

\section{The $\mathrm{SU}(2)$ case. I}

To understand the significance of Proposition 3, we will now spell out its meaning in the simplest case, where $\dot{K}=S U(2)$.

In doing explicit calculations, it is convenient to work with ordinary loops, rather than lifts in $\tilde{L} S U(2)$. Thus in this section, and the next, we will identify $S(1)$ with its projection to $L S U(2)$ (see Lemma 1). We will continue to denote this projection by $S(1)$.

In this case there is an (outer) automorphism of $\tilde{L} \dot{G}$ which interchanges the simple roots $\alpha_{0}$ and $\alpha_{1}$. At the level of loops, this automorphism is realized by conjugation by a multivalued loop,

$$
\operatorname{conj}\left(\left(\begin{array}{cc}
0 & i z^{1 / 2} \\
i z^{-1 / 2} & 0
\end{array}\right)\right):\left(\begin{array}{ll}
a & b \\
c & d
\end{array}\right) \rightarrow\left(\begin{array}{cc}
d & c z^{-1} \\
b z & a
\end{array}\right) .
$$


The root subgroup corresponding to $\alpha_{1}$ is $S L(2, \mathbb{C})$, the constants, and the root subgroup corresponding to $\alpha_{0}$ is the image of $S L(2, \mathbb{C})$ under this automorphism (see chapter 5 of [11]).

In [11] there is a relatively explicit realization of the groups $\tilde{L} S U(2, \mathbb{C})$ and $\tilde{L} S L(2, \mathbb{C})$. In this approach a loop $g \in L S L(2, \mathbb{C})$ is identified with a multiplication operator on $\mathcal{H}=L^{2}\left(S^{1}, \mathbb{C}^{2}\right)$. Relative to the Hardy polarization $\mathcal{H}=\mathcal{H}_{+} \oplus \mathcal{H}_{-}$,

$$
g=\left(\begin{array}{ll}
A & B \\
C & D
\end{array}\right)
$$

where $A$ (or $D$ ) is the classical Toeplitz operator and $C$ (or $B$ ) is the classical Hankel operator associated to $g$. The extension $\tilde{L} S L(2, \mathbb{C}) \rightarrow L S L(2, \mathbb{C})$ is the $\mathbb{C}^{*}$-bundle associated to the pullback of the determinant line bundle, relative to the mapping

$$
L S L(2, \mathbb{C}) \rightarrow \operatorname{Fred}\left(\mathcal{H}_{+}\right): g \rightarrow A(g) .
$$

The holomorphic function $\sigma_{0}$ on $\tilde{L} S L(2, \mathbb{C})$ is, viewed as a section of a line bundle, 'det $A(\tilde{g})$ '.

Suppose that $g \in L S U(2)$, and $\tilde{g} \in \tilde{L} S U(2)$ is a lift, which is uniquely determined up to multiplication by an element of the unitary center $\exp (i \mathbb{R} c)$. Then

$$
\left|\sigma_{0}(\tilde{g})\right|^{2}=\left|\sigma_{0}\right|^{2}(g)=\operatorname{det} A(g)^{*} A(g)=\operatorname{det}\left(1+Z(g)^{*} Z(g)\right)^{-1},
$$

where $Z=C A^{-1}$, and

$$
\left|\sigma_{1}\right|^{2}(g)=\left|\sigma_{0}\right|^{2}\left(\left(\begin{array}{cc}
z^{1 / 2} & 0 \\
0 & z^{-1 / 2}
\end{array}\right) g\left(\begin{array}{cc}
z^{-1 / 2} & 0 \\
0 & z^{1 / 2}
\end{array}\right)\right) .
$$

The simple reflections corresponding to the simple roots $\alpha_{0}$ and $\alpha_{1}$ are represented by the group elements

$$
s_{0}=\left(\begin{array}{cc}
0 & i z^{-1} \\
i z & 0
\end{array}\right), \quad \text { and } \quad s_{1}=\left(\begin{array}{cc}
0 & i \\
i & 0
\end{array}\right)
$$

respectively. We denote their images in the Weyl group by $\bar{s}_{i}$. The Weyl group (the affine Weyl group of $(\dot{\mathfrak{g}}, \dot{\mathfrak{h}}))$ has the structure

$$
W=\mathbb{Z}_{2} \bar{s}_{0} \propto \mathbb{Z}\left(\bar{s}_{0} \bar{s}_{1}\right)=\mathbb{Z}_{2} \bar{s}_{1} \propto \mathbb{Z}\left(\bar{s}_{0} \bar{s}_{1}\right)=\dot{W} \propto \operatorname{Hom}(\mathbb{T}, \dot{T}) .
$$

Minimal factorizations in the Weyl group must simply alternate the $\bar{s}_{i}$. This leads to two possible infinite minimal sequences of simple roots, the two possibilities depending upon whether one begins with $\alpha_{0}$ or $\alpha_{1}$. These are equivalent via the automorphism above. In the following theorem we will spell out Proposition 3 for the first possibility.

Theorem 5. Let $w_{1}=s_{0}, w_{2}=s_{1} s_{0}, w_{3}=s_{0} s_{1} s_{0}, \ldots$ Then for $n>0$,

(a)

$$
N^{-} \cap w_{n}^{-1} N^{+} w_{n}=\left\{l=\left(\begin{array}{cc}
1 & \sum_{j=1}^{n} x_{j} z^{-j} \\
0 & 1
\end{array}\right): x_{j} \in \mathbb{C}\right\} .
$$

(b) For the diffeomorphisms

$$
\mathbb{C}^{n} \rightarrow \mathbb{C}^{n}:\left(\zeta_{1}, \ldots, \zeta_{n}\right) \rightarrow x^{(n)}=\sum_{j=1}^{n} x_{j}^{(n)}\left(\zeta_{1}, \ldots, \zeta_{n}\right) z^{-j}
$$


arising from the isomorphism in part (1) of Proposition 3, and the parameterization in part (a),

$$
x_{j}^{(n)}\left(\zeta_{1}, \ldots, \zeta_{n}\right)=x_{j}^{(N)}\left(\zeta_{1}, \ldots, \zeta_{n}, 0, \ldots, 0\right),
$$

$n<N$ (hence we will often suppress the superscript), and

$$
x_{j}^{(n)}\left(\zeta_{1}, \ldots, \zeta_{n}\right)=x_{1}\left(\zeta_{j}, \ldots, \zeta_{n}, 0, \ldots\right) .
$$

(c) In terms of the correspondence of $\zeta$ with $g \in L S U(2)$ and $l \in N^{-}$, arising from the isomorphism in part (1) of Proposition 3,

$$
\left|\sigma_{0}\right|^{2}(g)=\frac{1}{\operatorname{det}\left(1+B\left(\sum_{1}^{n} x_{j} z^{j}\right) B\left(\sum_{1}^{n} x_{j} z^{j}\right)^{*}\right)}=\prod_{1}^{n} \frac{1}{\left(1+\left|\zeta_{j}\right|^{2}\right)^{j}}
$$

and

$$
\left|\sigma_{1}\right|^{2}(g)=\frac{1}{\operatorname{det}\left(1+B\left(\sum_{1}^{n-1} x_{j+1} z^{j}\right) B\left(\sum_{1}^{n-1} x_{j+1} z^{j}\right)^{*}\right)}=\prod_{1}^{n-1} \frac{1}{\left(1+\left|\zeta_{j+1}\right|^{2}\right)^{j-1}},
$$

where $B(\cdot)$ is defined as in Theorem 1. In particular

$$
\frac{\left|\sigma_{0}\right|}{\left|\sigma_{1}\right|}=\prod_{1}^{n} \frac{1}{\left(1+\left|\zeta_{j}\right|^{2}\right)^{1 / 2}}
$$

(d) More generally, for $0 \leq l<n$,

$$
\operatorname{det}\left(1+B\left(\sum_{1}^{n-l} x_{j+l} z^{j}\right) B\left(\sum_{1}^{n-l} x_{j+l} z^{j}\right)^{*}\right)=\prod_{1}^{n-l}\left(1+\left|\zeta_{j+l}\right|^{2}\right)^{j} .
$$

(e)

$$
\prod_{j=1}^{n} d \lambda\left(x_{j}\right)=\left(1+\left|\zeta_{2}\right|^{2}\right)^{2}\left(1+\left|\zeta_{2}\right|^{2}\right)^{4} \cdots\left(1+\left|\zeta_{n}\right|^{2}\right)^{2(n-1)} \prod_{j=1}^{n} d \lambda\left(\zeta_{j}\right) .
$$

Proof. Part (a) is a direct calculation. If $n=2 m$

$$
w_{n}=\left(s_{1} s_{0}\right)^{m}=(-1)^{m}\left(\begin{array}{cc}
z & 0 \\
0 & z^{-1}
\end{array}\right)^{m} .
$$

Thus if $u=\left(\begin{array}{ll}a & b \\ c & d\end{array}\right) \in N^{+}$, then

$$
w_{n}^{-1} u w_{n}=\left(\begin{array}{cc}
a & z^{-2 m} b \\
z^{2 m} c & d
\end{array}\right) \in N^{-}
$$

implies $a=d=1, c=0$, and $b=\sum_{0}^{2 m-1} b_{j} z^{j}$. This implies (a) when $n$ is even. The odd case is similar.

Before taking on the other parts of the Theorem, we need to understand what part (a) says in terms of the isomorphism of part (b) of Conjecture 1. It is straightforward to calculate that

$$
w_{j-1}^{-1} i_{\gamma_{j}}\left(k\left(\zeta_{j}\right)\right) w_{j-1}=a\left(\zeta_{j}\right)\left(\begin{array}{cc}
1 & \zeta_{j} z^{-j} \\
-\bar{\zeta}_{j} z^{j} & 1
\end{array}\right) .
$$


This implies that

$$
g=w_{n-1}^{-1} i_{\gamma_{n}}\left(k\left(\zeta_{n}\right)\right) w_{n-1} \cdots i_{\gamma_{1}}\left(k\left(\zeta_{1}\right)\right)=a\left(\zeta_{n}\right)\left(\begin{array}{cc}
1 & \zeta_{n} z^{-n} \\
-\bar{\zeta}_{n} z^{n} & 1
\end{array}\right) \cdots a\left(\zeta_{1}\right)\left(\begin{array}{cc}
1 & \zeta_{1} z^{-1} \\
-\bar{\zeta}_{1} z & 1
\end{array}\right) .
$$

If we write

$$
g=a\left(\zeta_{n}\right) \cdots a\left(\zeta_{1}\right)\left(\begin{array}{cc}
\alpha_{n} & \beta_{n} \\
\gamma_{n} & \delta_{n}
\end{array}\right)
$$

then there is a recursion relation

$$
\left(\begin{array}{c}
\beta_{n+1} \\
\delta_{n+1}
\end{array}\right)=\left(\begin{array}{cc}
1 & \zeta_{n} z^{-n-1} \\
-\bar{\zeta}_{n} z^{n+1} & 1
\end{array}\right)\left(\begin{array}{c}
\beta_{n} \\
\delta_{n}
\end{array}\right)
$$

In terms of the isomorphism in part (1) of Proposition 3, part (a) implies that

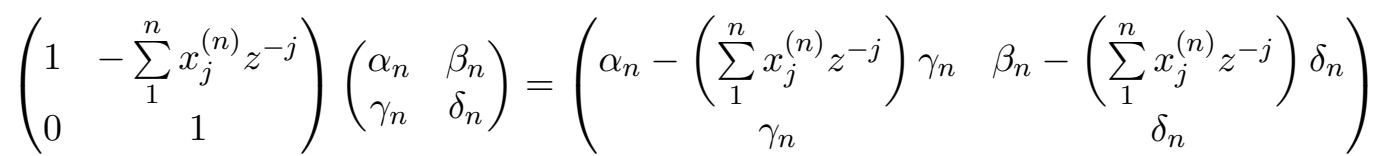

is an (entire) holomorphic function of $z$. In particular $\gamma_{n}$ and $\delta_{n}$ must be holomorphic functions of $z$, and

$$
\sum_{1}^{n} x_{j}^{(n)}\left(\zeta_{1}, \ldots, \zeta_{n}\right) z^{-j}=\left(\delta_{n}^{-1} \beta_{n}\right)_{-},
$$

where $(\cdot)_{-}$denotes the singular part (at $z=0$ ). The holomorphicity of (6.5) can be checked directly as follows. The recursion relation (6.4) shows that $\delta_{n}$ is of the form $1+\sum_{1}^{n} d_{j} z^{j}$, and $\beta_{n}$ is of the form $\sum_{1}^{n} b_{j} z^{-j}$. Since $\gamma_{n}=-\beta_{n}^{*}$ on $S^{1}$, this shows $\gamma_{n}$ and $\delta_{n}$ are holomorphic functions

of $z$. It also shows the $x_{j}^{(n)}$ are well-defined by (6.6). The relation (6.6) implies the $(1,2)$ entry of $(6.5)$ is holomorphic. Also (6.6) implies the $(1,1)$ entry of $(6.5)$ is of the form

$$
\begin{aligned}
& \alpha_{n}-\delta_{n}^{-1} \beta_{n} \gamma_{n}+\text { holomorphic }=\delta_{n}^{-1}\left(\alpha_{n} \delta_{n}-\beta_{n} \gamma_{n}\right)+\text { hol. } \\
& \left.\quad=\delta_{n}^{-1}\left(\alpha_{n} \alpha_{n}^{*}+\beta_{n} \beta_{n}^{*}\right)+\text { hol. }=\text { (const }\right) \delta_{n}^{-1}+\text { hol. }=\text { holomorphic. }
\end{aligned}
$$

We now consider part (b). We will need several Lemmas.

\section{Lemma 2.}

(a) The $x^{(n)}$ satisfy the recursion relation

$$
\begin{aligned}
x^{(n+1)} & =\left(\left(x^{(n)}+\zeta_{n+1} z^{-n-1}\right) \sum_{p=0}^{n}\left(\bar{\zeta}_{n+1} x^{(n)} z^{n+1}\right)^{p}\right)_{-} \\
& =\left(\sum_{p=0}^{n} \bar{\zeta}_{n+1}^{p}\left(x^{(n)}\right)^{p+1}\left(1+\left|\zeta_{n+1}\right|^{2}\right) z^{p(n+1)}\right)_{-}+\zeta_{n+1} z^{-n-1} .
\end{aligned}
$$

(b) $x^{(n)}$ can be replaced by $x^{(n)}+h(z)$, where $h(z)$ is a holomorphic function, without changing the recursion.

Proof. The recursion relation (6.4) and (6.6) imply that $x^{(n+1)}$ is the singular part of

$$
\left(\beta_{n}+\delta_{n} \zeta_{n+1} z^{-n-1}\right)\left(\delta_{n}-\bar{\zeta}_{n+1} z^{n+1} \beta_{n}\right)^{-1}=\left(\beta_{n} \delta_{n}^{-1}+\zeta_{n+1} z^{-n-1}\right)\left(1-\bar{\zeta}_{n+1} \beta_{n} \delta_{n}^{-1} z^{n+1}\right)^{-1}
$$




$$
=\left(\beta_{n} \delta_{n}^{-1}+\zeta_{n+1} z^{-n-1}\right) \sum_{p=0}^{\infty}\left(\bar{\zeta}_{n+1} x^{(n)} z^{n+1}\right)^{p} .
$$

Since $x^{(n)} z^{n+1}$ is $O(z)$, the singular part of (6.9) equals the right hand side of (6.7).

We now rewrite the right hand side of (6.7) as

$$
\begin{aligned}
\left(\left(x^{(n)}+\zeta_{n+1} z^{-n-1}\right) \sum_{p=0}^{n}\left(\bar{\zeta}_{n+1} x^{(n)} z^{n+1}\right)^{p}\right)_{-} \\
=\left(\sum_{p=0}^{n} \bar{\zeta}_{n+1}^{p}\left(x^{(n)}\right)^{p+1} z^{p(n+1)}+\zeta_{n+1} z^{-n-1}+\sum_{p=1}^{n}\left|\zeta_{n+1}\right|^{2} \bar{\zeta}_{n+1}^{p-1}\left(x^{(n)}\right)^{p} z^{(p-1)(n+1)}\right)_{-} \\
\\
+\left(\sum_{p=0}^{n} \bar{\zeta}_{n+1}^{p}\left(x^{(n)}\right)^{p+1} z^{p(n+1)}+\zeta_{n+1} z^{-n-1}+\sum_{p=1}^{n}\left|\zeta_{n+1}\right|^{2} \bar{\zeta}_{n+1}^{p}\left(x^{(n)}\right)^{p+1} z^{p(n+1)}\right)_{-} \\
\quad+\left(\sum_{p=0}^{n} \bar{\zeta}_{n+1}^{p}\left(x^{(n)}\right)^{p+1}\left(1+\left|\zeta_{n+1}\right|^{2}\right) z^{p(n+1)}\right)_{-}+\zeta_{n+1} z^{-n-1} .
\end{aligned}
$$

This completes the proof of part (a).

Part (b) is obvious.

For small $n$ the recursion implies $x^{(1)}=\zeta_{1} z^{-1}$,

$$
\begin{aligned}
x^{(2)}= & \zeta_{1}\left(1+\left|\zeta_{2}\right|^{2}\right) z^{-1}+\zeta_{2} z^{-2}, \\
x^{(3)}= & \left(\zeta_{1}\left(1+\left|\zeta_{2}\right|^{2}\right)\left(1+\left|\zeta_{3}\right|^{2}\right)+\zeta_{2}\left(1+\left|\zeta_{3}\right|^{2}\right) \zeta_{2} \bar{\zeta}_{3}\right) z^{-1}+\zeta_{2}\left(1+\left|\zeta_{3}\right|^{2}\right) z^{-2}+\zeta_{3} z^{-3}, \\
x^{(4)}= & \left(\zeta_{1} \prod_{2}^{4}\left(1+\left|\zeta_{j}\right|^{2}\right)+\zeta_{2} \prod_{3}^{4}\left(1+\left|\zeta_{j}\right|^{2}\right)\left(\zeta_{2} \bar{\zeta}_{3}+2 \zeta_{3} \bar{\zeta}_{4}\right)+\zeta_{3}\left(1+\left|\zeta_{4}\right|^{2}\right)\left(\zeta_{3} \bar{\zeta}_{4}\right)^{2}\right) z^{-1} \\
& +\left(\zeta_{2} \prod_{3}^{4}\left(1+\left|\zeta_{j}\right|^{2}\right)+\zeta_{3}\left(1+\left|\zeta_{4}\right|^{2}\right) \zeta_{3} \bar{\zeta}_{4}\right) z^{-2}+\zeta_{3}\left(1+\left|\zeta_{4}\right|^{2}\right) z^{-3}+\zeta_{4} z^{-4}
\end{aligned}
$$

Lemma 3. $y^{(n)}=\left(z x^{(n+1)}\right)_{-}$depends only on $\zeta_{2}, \ldots, \zeta_{n+1}$, and satisfies the same recursion as $x^{(n)}$, with the shifted variables $\zeta_{2}, \ldots$ in place of $\zeta_{1}, \ldots$

Proof. For small $n$ the formulas above show that $y^{(n)}$ does not depend on $\zeta_{1}$. By Lemma 2

$$
\begin{aligned}
y^{(n)} & =\left(z\left(\left(x^{(n)}+\zeta_{n+1} z^{-n-1}\right) \sum_{p=0}^{n}\left(\bar{\zeta}_{n+1} x^{(n)} z^{n+1}\right)^{p}\right)_{-}\right) \\
& =\left(\left(z x^{(n)}+\zeta_{n+1} z^{-n}\right) \sum_{p=0}^{n}\left(\bar{\zeta}_{n+1} z x^{(n)} z^{n}\right)^{p}\right)_{-} \\
& =\left(\left(y^{(n-1)}+\zeta_{n+1} z^{-n}\right) \sum_{p=0}^{n}\left(\bar{\zeta}_{n+1} y^{(n-1)} z^{n}\right)^{p}\right)_{-} .
\end{aligned}
$$

This establishes the recursion and induction implies $y^{(n)}$ does not depend on $\zeta_{1}$. 
We can now complete the proof of part (b). Lemma 3 implies that

$$
\left(z x^{(n+1)}\right)_{-}=\sum_{2}^{n+1} x_{j}^{(n+1)}\left(\zeta_{1}, \ldots, \zeta_{n+1}\right) z^{-j+1}=\sum_{1}^{n} x_{i}^{(n)}\left(\zeta_{2}, \ldots, \zeta_{n}\right) z^{-i}
$$

This implies that for $j>1$,

$$
x_{j}^{(n+1)}\left(\zeta_{1}, \ldots, \zeta_{n+1}\right)=x_{j-1}^{(n)}\left(\zeta_{2}, \ldots, \zeta_{n+1}\right) .
$$

By induction this implies part (b). This also implies

$$
x^{(n+1)}=x_{1}^{(n+1)} z^{-1}+x^{(n)}\left(\zeta_{2}, \ldots, \zeta_{n+1}\right) z^{-1} .
$$

For future reference, note that there is a recursion for $x_{1}$ of the form

$$
\begin{aligned}
& x_{1}\left(\zeta_{1}, \ldots, \zeta_{n+1}\right)=x_{1}\left(\zeta_{1}, \ldots, \zeta_{n}\right)\left(1+\left|\zeta_{n+1}\right|^{2}\right) \\
& \quad+\left(\sum_{i+j=n+2} x_{1}\left(\zeta_{i}, \ldots, \zeta_{n}\right) x_{1}\left(\zeta_{j}, \ldots, \zeta_{n}\right)\right) \bar{\zeta}_{n+1}\left(1+\left|\zeta_{n+1}\right|^{2}\right) \\
& \quad+\left(\sum_{i+j+k=2 n+3} x_{1}\left(\zeta_{i}, \ldots, \zeta_{n}\right) x_{1}\left(\zeta_{j}, \ldots, \zeta_{n}\right) x_{1}\left(\zeta_{k}, \ldots, \zeta_{n}\right)\right) \bar{\zeta}_{n+1}^{2}\left(1+\left|\zeta_{n+1}\right|^{2}\right)+\cdots
\end{aligned}
$$

It would be highly desirably to find a closed form solution of this recursion for $x_{1}$.

We now consider part (c). For $g$ as in part (c), consider the Riemann-Hilbert factorization $g=g_{-} g_{0} g_{+}$, where

$$
g_{-}=\left(\begin{array}{cc}
1 & x \\
0 & 1
\end{array}\right), \quad g_{0}=\left(\begin{array}{cc}
a_{0} & b_{0} \\
0 & a_{0}^{-1}
\end{array}\right) \in S L(2, \mathbb{C}),
$$

and $g_{+} \in H^{0}(D, 0 ; S L(2, \mathbb{C}), 1)$. Then

$$
Z(g)=C(g) A(g)^{-1}=C\left(g_{-}\right) A\left(g_{0} g_{+}\right) A\left(g_{0} g_{+}\right)^{-1} A\left(g_{-}\right)=Z\left(g_{-}\right) .
$$

(For use in the next paragraph, note that this calculation does not depend on the specific form of $g_{0}$.) Let $\epsilon_{1}, \epsilon_{2}$ denote the standard basis for $\mathbb{C}^{2}$. As in [11], consider the ordered basis $\ldots, \epsilon_{1} z^{j+1}, \epsilon_{2} z^{j+1}, \epsilon_{1} z^{j}, \ldots, j \in \mathcal{Z}$, for $\mathcal{H}$. This basis is compatible with the Hardy polarization of $\mathcal{H}$. We claim that

$$
Z\left(g_{-}\right)=C\left(g_{-}\right)=\left(\begin{array}{cccccccccc}
. & 0 & x_{n} & . & 0 & x_{3} & 0 & x_{2} & 0 & x_{1} \\
. & 0 & 0 & . & 0 & 0 & 0 & 0 & 0 & 0 \\
\cdot & . & . & & & x_{4} & 0 & x_{3} & 0 & x_{2} \\
& & & & & 0 & 0 & 0 & 0 & 0 \\
\cdot & & & & & & & & 0 & x_{3} \\
\cdot & & . & & & . & . & & & \\
. & & & & & . & & & 0 & x_{n} \\
0 & 0 & 0 & 0 & . & . & & 0 & 0 & 0
\end{array}\right) .
$$

Let $P_{ \pm}$denote the orthogonal projections associated to the Hardy splitting of $\mathcal{H}$. For example

$$
P_{+}\left(f=\sum f_{k} z^{k}\right)=\sum_{k \geq 0} f_{k} z^{k}
$$


Suppose that $\left(\begin{array}{l}f_{1} \\ f_{2}\end{array}\right) \in \mathcal{H}^{+}$. Then

$$
\begin{aligned}
& C\left(g_{-}\right) A\left(g_{-}\right)^{-1}\left(\begin{array}{l}
f_{1} \\
f_{2}
\end{array}\right)=P_{-} g_{-} P_{+} g_{-}^{-1}\left(\begin{array}{l}
f_{1} \\
f_{2}
\end{array}\right)=P_{-} g_{-}\left(\begin{array}{c}
f_{1}-P_{+}\left(x f_{2}\right) \\
f_{2}
\end{array}\right), \\
& P_{-}\left(\begin{array}{c}
f_{1}+P_{+}\left(x f_{2}\right)+x f_{2} \\
f_{2}
\end{array}\right)=\left(\begin{array}{c}
P_{-}\left(x f_{2}\right) \\
0
\end{array}\right)=C\left(g_{-}\right)\left(\begin{array}{l}
f_{1} \\
f_{2}
\end{array}\right) .
\end{aligned}
$$

This is the first part of the claim. For the second part one simply calculates directly, using the simple form for $g_{-}$.

Comparing (6.15) with (1.4) proves the first part of (c).

Using the factorization

$$
g=\left(\begin{array}{ll}
1 & x \\
0 & 1
\end{array}\right) g_{0} g_{+}
$$

and the specific form of $g_{0}$, it is clear that

$$
g_{-}\left(\left(\begin{array}{cc}
z^{1 / 2} & 0 \\
0 & z^{-1 / 2}
\end{array}\right) g\left(\begin{array}{cc}
z^{-1 / 2} & 0 \\
0 & z^{1 / 2}
\end{array}\right)\right)=\left(\begin{array}{cc}
1 & x^{\prime} \\
0 & 1
\end{array}\right)
$$

where $x^{\prime}=(z x)_{-}=x_{2} z^{-1}+\cdots+x_{n} z^{-(n-1)}$. We now use (6.3) and (6.15) to prove the second part of (c).

Part (d) follows from (c). Part (e) can be read off from Lemma 2 (see (6.10)-(6.12)), or from part (2) of Proposition 3.

We are now in a position to prove Theorem 1 at the end of the Introduction.

Proof. By parts (d) and (e) of Theorem 5,

$$
\begin{aligned}
\int \frac{1}{\prod_{l=0}^{n-1} \operatorname{det}\left(1+B\left(\sum_{j=1}^{n-l} x_{l+j} z^{j}\right) B_{n}\left(\sum_{j=1}^{n-l} x_{l+j} z^{j}\right)^{*}\right)^{p_{l}}} d \lambda\left(x_{1}, \ldots, x_{n}\right) \\
=\int\left(\prod_{l=0}^{n-1} \prod_{j=1}^{n-l}\left(1+\left|\zeta_{l+j}\right|^{2}\right)^{-j p_{l}}\right) \prod_{j=1}^{n}\left(1+\left|\zeta_{j}\right|^{2}\right)^{-2(j-1)} d \lambda\left(\zeta_{j}\right) \\
=\int\left(1+\left|\zeta_{1}\right|^{2}\right)^{-p_{1}} d \lambda\left(\zeta_{1}\right) \int\left(1+\left|\zeta_{2}\right|^{2}\right)^{2-\left(2 p_{1}+p_{2}\right)} d \lambda\left(\zeta_{2}\right) \cdots \\
\quad \ldots \int\left(1+\left|\zeta_{n}\right|^{2}\right)^{2 n-2-\left(n p_{1}+\cdots+p_{n}\right)} d \lambda\left(\zeta_{n}\right) \\
=\pi^{n} \frac{1}{p_{1}-1} \frac{1}{2 p_{1}+p_{2}-3} \cdots \frac{1}{n p_{1}+(n-1) p_{2}+\cdots+p_{n}-(2 n-1)}
\end{aligned}
$$

\section{The SU(2) case. II}

This is a continuation of Section 6 . We first consider the limit $n \rightarrow \infty$, in the context of Theorem 5. From the point of view of analysis, this limit is naturally related to the critical exponent $s=1 / 2$ for the circle. We secondly show that there is a global factorization of the momentum mapping for $(S(1), \omega)$, extending the formulas in (c) of Theorem 5 . As in Section 6 , we will continue to view $S(1)$ as a submanifold of $L S U(2)$, rather than $\tilde{L} S U(2)$. 
As in (6.16), we let $P_{ \pm}$denote the orthogonal projections associated to the Hardy splitting of $L^{2}\left(S^{1}\right)$. Given $f=\sum f_{n} z^{n}$, we will write $f^{*}=\sum \bar{c}_{n} z^{-n}$. If we simply write $z$ for the multiplication operator corresponding to $z$, then

$$
(\cdot)^{*} \circ P_{-}=z \circ P_{+} \circ z^{-1} \circ(\cdot)^{*}
$$

and

$$
(\cdot)^{*} \circ P_{+}=z \circ P_{-} \circ z^{-1} \circ(\cdot)^{*} .
$$

For a function $F \in L^{\infty}\left(S^{1}\right)$, viewed as a bounded multiplication operator on $L^{2}\left(S^{1}\right)$, we will write $A(F)=P_{+} F P_{+}$, and so on, as in (6.2).

Suppose

$$
l=\left(\begin{array}{ll}
1 & x \\
0 & 1
\end{array}\right), \quad \text { where } \quad x=\sum_{1}^{n} x_{j} z^{-j} .
$$

There exists a unique $g \in L_{\mathrm{pol}} S U(2)$ with unique triangular factorization $g=l a(g)^{h_{1}} u$ where $a(g)=\left|\sigma_{1}\right|(g) /\left|\sigma_{0}\right|(g)$, and

$$
\left|\sigma_{0}\right|^{2}=\operatorname{det}\left(1+C(x)^{*} C(x)\right)^{-1}, \quad\left|\sigma_{1}\right|^{2}=\operatorname{det}\left(1+C(z x)^{*} C(z x)\right)^{-1}
$$

(see part (c) of Theorem 5).

Lemma 4. The triangular factorization of $g \in L_{\mathrm{pol}} S U(2)$ is given by

$$
g=\left(\begin{array}{ll}
1 & x \\
0 & 1
\end{array}\right)\left(\begin{array}{cc}
a & 0 \\
0 & a^{-1}
\end{array}\right)\left(\begin{array}{cc}
1+\sum_{1}^{n-1} \alpha_{j} z^{j} & \sum_{0}^{n-2} \beta_{j} z^{j} \\
\sum_{1}^{n} \gamma_{j} z^{j} & 1+\sum_{1}^{n-1} \delta_{j} z^{j}
\end{array}\right)
$$

where

$$
\begin{aligned}
& \gamma=-\left(\left(1+C(z x) C(z x)^{*}\right)^{-1}(x)\right)^{*}, \quad \delta^{*}=C(x) \gamma, \\
& 1+\alpha=\frac{1}{a^{2}}(1-A(x) \gamma), \quad \beta=-\frac{1}{a^{2}} A(x)(1+\delta) .
\end{aligned}
$$

Proof. Because

$$
g=\left(\begin{array}{cc}
a(1+\alpha)+a^{-1} x \gamma & a \beta+x a^{-1}(1+\delta) \\
a^{-1} \gamma & a^{-1}(1+\delta)
\end{array}\right)
$$

has values in $S U(2)$ (as a function of $z \in S^{1}$ ),

$$
a^{2}(1+\alpha)+x \gamma=1+\delta^{*}, \quad \text { and } \quad a^{2} \beta+x(1+\delta)=-\gamma^{*} .
$$

The first equation can be expressed in operator language as

$$
A(x) \gamma=1-a^{2}(1+\alpha), \quad C(x) \gamma=\delta^{*} .
$$

The second equation is equivalent to

$$
A(x)(1+\delta)=-a^{2} \beta, \quad C(x)(\delta)=-x-\gamma^{*} .
$$

We can solve for $\delta$, using the second equation of (7.2),

$$
(\cdot)^{*} \circ C(x)(\gamma)=z B\left(z^{-1} x^{*}\right)\left(\gamma^{*}\right)=\delta
$$


The second equation of (7.3) now implies

$$
C(x) z B\left(z^{-1} x^{*}\right)\left(\gamma^{*}\right)=-x-\gamma^{*}
$$

which is equivalent to

$$
\left(1+C(z x) C(z x)^{*}\right)\left(\gamma^{*}\right)=-x
$$

The Lemma follows from these equations.

Let $W^{1 / 2}$ denote the Sobolev space of (Lebesgue equivalence classes of) functions having half of a derivative, i.e. if $f=\sum f_{j} z^{j}$, then $\sum j\left|f_{j}\right|^{2}<\infty$. A class in $W^{1 / 2}$ is not in general represented by a continuous function. Despite this, $W^{1 / 2}\left(S^{1}, S U(2)\right)$ is a connected topological group (homotopy equivalent to $L S U(2)$; see [1]), and it is the natural domain for the basic factorization theorems in the theory of loop groups (see Chapter 8 of [11]). We let $\mathbb{S}(1)$ denote the completion of $S(1)$ in $W^{1 / 2}\left(S^{1}, S U(2)\right)$.

Theorem 6. By taking a limit as $n \rightarrow \infty$ in Theorem 5, we obtain bijective correspondences among the following three sets

(a) $\left\{\zeta=\left(\zeta_{1}, \zeta_{2}, \ldots\right): \sum_{1}^{\infty} j\left|\zeta_{j}\right|^{2}<\infty\right\}$.

(b) $x=\sum_{1}^{\infty} x_{j} z^{-j} \in W^{1 / 2}$, where (as in (b) of Theorem 5)

$$
x_{j}\left(\zeta_{1}, \ldots\right)=\lim _{n \rightarrow \infty} x_{1}\left(\zeta_{j}, \ldots, \zeta_{n}\right)
$$

and conversely

$$
\zeta_{j}\left(x_{1}, \ldots\right)=\lim _{n \rightarrow \infty} \zeta_{1}\left(x_{j}, \ldots, x_{n}\right) .
$$

(c)

$$
\left\{g=\left(\begin{array}{cc}
d(z)^{*} & -c(z)^{*} \\
c(z) & d(z)
\end{array}\right) \in \mathbb{S}(1): c(z), d(z) \in H^{0}(\Delta), c(0)=0\right\},
$$

where

$$
g=\lim _{n \rightarrow \infty} a\left(\zeta_{n}\right)\left(\begin{array}{cc}
1 & \zeta_{n} z^{-n} \\
-\bar{\zeta}_{n} z^{n} & 1
\end{array}\right) \cdots a\left(\zeta_{1}\right)\left(\begin{array}{cc}
1 & \zeta_{1} z^{-1} \\
-\bar{\zeta}_{1} z & 1
\end{array}\right)
$$

$a\left(\zeta_{j}\right)=\left(1+\left|\zeta_{j}\right|^{2}\right)^{-1 / 2}, g$ has triangular factorization

$$
g=\left(\begin{array}{cc}
1 & \sum_{1}^{\infty} x_{j} z^{-j} \\
0 & 1
\end{array}\right)\left(\begin{array}{cc}
\frac{\left|\sigma_{1}\right|(g)}{\left|\sigma_{0}\right|(g)} & 0 \\
0 & \frac{\left|\sigma_{0}\right|(g)}{\left|\sigma_{1}\right|(g)}
\end{array}\right) u
$$

and the entries of $u$ are given by the same formulas as in Lemma 4.

Note that for $k>0 g=\left(\begin{array}{cc}z^{-k} & 0 \\ 0 & z^{k}\end{array}\right)$ has a second row which is holomorphic in the disk, but $g$ is not in $S(1)$. Thus in part (c) it is necessary to require that $g \in \mathbb{S}(1)$. 
Proof. By Theorem 5, for $\zeta$ with a finite number of terms,

$$
\prod\left(1+\left|\zeta_{j}\right|^{2}\right)^{j}=\operatorname{det}\left(1+C(x) C(x)^{*}\right)
$$

and obviously

$$
\operatorname{tr}\left(C(x) C(x)^{*}\right)=\sum j\left|x_{j}\right|^{2} .
$$

For an arbitrary sequence $\zeta$, the product on the LHS of (7.7) is finite iff $\sum_{1}^{\infty} j\left|\zeta_{j}\right|^{2}<\infty$. Similarly, for an arbitrary $x$, the determinant is finite iff (7.8) is finite. Thus given $\zeta$ as in part (a), the partial sums for the series representing $x$ will have limits in $W^{1 / 2}$. To understand why there is a unique limit, and to prove the other statements in part (b), recall that the recursion relation (6.13) implies that $x_{1}$ has a series expansion, with nonnegative integer coefficients, in terms of the variables $\zeta_{j}, \bar{\zeta}_{j}$, of the form

$$
\begin{aligned}
x_{1}\left(\zeta_{1}, \ldots\right)= & \zeta_{1} \prod_{j=2}^{\infty}\left(1+\left|\zeta_{j}\right|^{2}\right)+\zeta_{2} \prod_{j=3}^{\infty}\left(1+\left|\zeta_{j}\right|^{2}\right)\left(\zeta_{2} \bar{\zeta}_{3}+2 \zeta_{3} \bar{\zeta}_{4}+\cdots\right) \\
& +\zeta_{3} \prod_{j=4}^{\infty}\left(1+\left|\zeta_{j}\right|^{2}\right)\left(\left(\zeta_{3} \bar{\zeta}_{4}\right)^{2}+\cdots\right)+\cdots
\end{aligned}
$$

Since $\left|x_{1}\right|^{2}$ is dominated by (7.8), this series will converge absolutely. Thus $x_{1}$ is a well-defined function of $\zeta$. The relation (7.4) follows from (b) of Theorem (5). Thus all the $x_{j}$, and hence also $x \in W^{1 / 2}$, are uniquely determined by $\zeta$, assuming $\sum j\left|\zeta_{j}\right|^{2}<\infty$.

Because

$$
\prod_{j=k}^{\infty}\left(1+\left|\zeta_{j}\right|^{2}\right)=\frac{\operatorname{det}\left(1+C\left(z^{k} x\right) C\left(z^{k} x\right)^{*}\right)}{\operatorname{det}\left(1+C\left(z^{k-1} x\right) C\left(z^{k-1} x\right)^{*}\right)}
$$

and the triangular nature of the relation between the $\zeta_{j}$ and the $x_{j}$, the map from the $\zeta$ to $x$ can be inverted, and the $\zeta_{j}$ will be expressible in terms of $\zeta_{1}$ as in (7.5). We have thus established that there is a bijective correspondence between the sets in (a) and (b).

Now suppose that we are given $x$ as in part (b). We claim that (7.1) and Lemma 4 imply that we can obtain a $g$ as in part (c), with triangular factorization as in (7.6). Because $x \in W^{1 / 2}$, $C(x)$ and $C(z x)$ are Hilbert-Schmidt operators (viewed as operators on $L^{2}\left(S^{1}\right)$ ), so that the formulas (7.1), and hence the formula for $a(g)$, make sense. The formulas for $\gamma$ and $\delta$ in Lemma 4 a priori show only that $\gamma$ and $\delta$ are $L^{2}\left(S^{1}\right)$ (not necessarily $W^{1 / 2}$ ), so that we obtain a $S U(2)$ loop $g$, expressed as in part (c), with $L^{2}$ entries. But as in $(6.14), Z(g)=Z\left(\left(\begin{array}{ll}1 & x \\ 0 & 1\end{array}\right)\right)$, and this is a Hilbert-Schmidt operator. Because $g \in L^{\infty}\left(S^{1}\right), A(g)$ is a bounded operator, and hence this implies that $C(g)$ is a Hilbert-Schmidt operator. Thus $g \in W^{1 / 2}$.

Conversely given $g$ as in part (c), it is obvious that $g$ has a triangular form as in (7.6), and this determines $x$. The equality

$$
\operatorname{det}|A(g)|^{2}=\operatorname{det}\left(1+C(x) C(x)^{*}\right)^{-1}
$$

implies that $x \in W^{1 / 2}$. Because the sets in (a) and (b) are in correspondence, this also determines $\zeta$. This completes the proof.

Recall that in Theorem 5 we considered the minimal sequence $\alpha_{0}, \alpha_{1}, \ldots$ By considering the sequence $\alpha_{1}, \alpha_{0}, \ldots$, or in other words, by applying the automorphism (6.1) which interchanges the two simple roots, the proceeding Theorem can be reformulated in the following way. 
Corollary 1. There are bijective correspondences among the following three sets

(a) $\left\{\eta=\left(\eta_{0}, \eta_{1}, \ldots\right): \sum_{0}^{\infty} j\left|\eta_{j}\right|^{2}<\infty\right\}$.

(b) $y=\sum_{0}^{\infty} y_{j} z^{-j} \in W^{1 / 2}$, where

$$
y_{j}\left(\eta_{0}, \ldots\right)=\lim _{n \rightarrow \infty} y_{0}\left(\eta_{j}, \ldots, \eta_{n}\right)
$$

and conversely

$$
\eta_{j}\left(y_{0}, \ldots\right)=\lim _{n \rightarrow \infty} \eta_{0}\left(y_{j}, \ldots, y_{n}\right) .
$$

(c)

$$
\left\{h=\left(\begin{array}{cc}
e(z) & b(z) \\
-b^{*}(z) & e(z)^{*}
\end{array}\right) \in \mathbb{S}(1): b(z), e(z) \in H^{0}(\Delta)\right\},
$$

where

$$
h=\lim _{n \rightarrow \infty} a\left(\eta_{n}\right)\left(\begin{array}{cc}
1 & \eta_{n} z^{n} \\
-\bar{\eta}_{n} z^{-n} & 1
\end{array}\right) \cdots a\left(\eta_{0}\right)\left(\begin{array}{cc}
1 & \eta_{0} \\
-\bar{\eta}_{0} & 1
\end{array}\right),
$$

and $h$ has triangular factorization of the form

$$
\begin{aligned}
& h=\left(\begin{array}{ll}
1 & 0 \\
y & 1
\end{array}\right)\left(\begin{array}{cc}
\left|\sigma_{1}\right|(h) & 0 \\
\left|\sigma_{0}\right|(h) & \frac{\left|\sigma_{0}\right|(h)}{\left|\sigma_{1}\right|(h)}
\end{array}\right)\left(\begin{array}{cc}
1+\sum_{1}^{\infty} \alpha_{j} z^{j} & \sum_{0}^{\infty} \beta_{j} z^{j} \\
\sum_{1}^{\infty} \gamma_{j} z^{j} & 1+\sum_{1}^{\infty} \delta_{j} z^{j}
\end{array}\right), \\
& \beta=-\left(1+C(y)^{*} C(y)\right)^{-1}\left(y^{*}\right), \\
& 1+\delta=a(h)^{2}(1-A(y) \beta), \quad \alpha^{*}=C(y) \beta,
\end{aligned}
$$

Moreover

$$
\left|\sigma_{0}\right|^{2}(h)=\prod_{j=0}^{\infty}\left(1+\left|\eta_{j}\right|^{2}\right)^{-j}, \quad\left|\sigma_{1}\right|^{2}(h)=\prod_{j=0}^{\infty}\left(1+\left|\eta_{j}\right|^{2}\right)^{-(j+1)}
$$

and

$$
a(h)=\frac{\left|\sigma_{1}\right|}{\left|\sigma_{0}\right|}=\prod\left(1+\left|\eta_{j}\right|^{2}\right)^{-1 / 2} .
$$

In the following statement we will continue to view $\mathbb{S}(1)$ as a subset of $W^{1 / 2}\left(S^{1}, S U(2)\right)$, but in the proof it will be necessary to consider lifts in the Kac-Moody extension (as in Lemma 1).

Theorem 7. Suppose that $\zeta, \chi$, and $\eta$ are sequences such that

$$
\sum_{j=1}^{\infty} j\left(\left|\zeta_{j}\right|^{2}+\left|\chi_{j}\right|^{2}+\left|\eta_{j}\right|^{2}\right)<\infty
$$

By slight abuse of notation, we identify $\chi$ with the function $\chi=\sum_{j=1}^{\infty} \chi_{j} z^{j}$. Let $g$ be defined as in (c) of Theorem 6 and $h$ as in (c) of Corollary 1.

(a) The product $h^{-1} e^{\left(\chi-\chi^{*}\right) h_{1}} g \in \mathbb{S}(1)$. 
(b) The mapping

$$
(\eta, \chi, \zeta): \sum j\left(\left|\eta_{j}\right|^{2}+\left|\chi_{j}\right|^{2}+\left|\zeta_{j}\right|^{2}\right)<\infty \rightarrow \mathbb{S}(1):(\eta, \chi, \zeta) \rightarrow h^{-1} e^{\left(\chi-\chi^{*}\right) h_{1}} g
$$

is injective.

(c)

$$
\begin{aligned}
& \left|\sigma_{0}\right|^{2}\left(h^{-1} e^{\left(\chi-\chi^{*}\right) h_{1}} g\right)=\prod_{j=1}\left(1+\left|\eta_{j}\right|^{2}\right)^{-j} \exp \left(-2 \sum_{j=1} j\left|\chi_{j}\right|^{2}\right) \prod_{j=1}\left(1+\left|\zeta_{j}\right|^{2}\right)^{-j}, \\
& \left|\sigma_{1}\right|^{2}\left(h^{-1} e^{\left(\chi-\chi^{*}\right) h_{1}} g\right)=\prod_{j=1}\left(1+\left|\eta_{j+1}\right|^{2}\right)^{-j} \exp \left(-2 \sum_{j=1} j\left|\chi_{j}\right|^{2}\right) \prod_{j=1}\left(1+\left|\zeta_{j+1}\right|^{2}\right)^{-j}, \\
& a^{2}\left(h^{-1} e^{\left(\chi-\chi^{*}\right) h_{1}} g\right)=\prod_{j=1} \frac{1+\left|\zeta_{j}\right|^{2}}{1+\left|\eta_{j}\right|^{2}} .
\end{aligned}
$$

Proof. For part (a), we do a calculation at the level of loops. If we write $h$ as in (7.9), then the triangular factorization of $h^{-1}$ is given by

$$
h^{-1}=l\left(h^{-1}\right)\left(\begin{array}{cc}
a\left(h^{-1}\right) & 0 \\
0 & a\left(h^{-1}\right)^{-1}
\end{array}\right)\left(\begin{array}{cc}
1 & y^{*} \\
0 & 1
\end{array}\right)
$$

where

$$
l\left(h^{-1}\right)=u(h)^{*}=\left(\begin{array}{cc}
1+\alpha^{*} & \gamma^{*} \\
\beta^{*} & 1+\delta^{*}
\end{array}\right) .
$$

Then

$$
h^{-1} e^{\left(\chi-\chi^{*}\right) h_{1}} g=l\left(h^{-1}\right) a\left(h^{-1}\right)^{h_{1}}\left(\begin{array}{cc}
1 & y^{*} \\
0 & 1
\end{array}\right) e^{\left(\chi-\chi^{*}\right) h_{1}}\left(\begin{array}{cc}
1 & x \\
0 & 1
\end{array}\right) a(g)^{h_{1}} u(g) .
$$

The main point of the proof is that the middle three factors are upper triangular, and we can find the (loop space) triangular factorization of the product with ease. Thus (7.10) equals

$$
\begin{aligned}
& l\left(h^{-1}\right) e^{-\chi^{*} h_{1}}\left(\begin{array}{cc}
1 & a\left(h^{-1}\right)^{2} P_{-}\left(y^{*} e^{2 \chi^{*}}+x e^{2 \chi}\right) \\
0 & 1
\end{array}\right) \\
& \quad \times a(h)^{h_{1}} a(g)^{h_{1}}\left(\begin{array}{cc}
1 & a(g)^{-2} P_{+}\left(y^{*} e^{2 \chi^{*}}+x e^{2 \chi}\right) \\
0 & 1
\end{array}\right) e^{\chi h_{1}} u(g) .
\end{aligned}
$$

This triangular form implies part (a) of the Theorem.

This calculation also implies that $a\left(h^{-1} e^{\left(\chi-\chi^{*}\right) h_{1}} g\right)=a\left(h^{-1}\right) a(g)$. Given that $a\left(h^{-1}\right)=$ $a\left(h^{*}\right)=a(h)$, and formulas we have already established for $a(g)$ and $a(h)$ (see Corollary 1 ), this also implies the third formula in part (c).

To obtain the first two formulas in part (c), we need to lift each of the factors, $h^{-1}$, the torus-valued loop, and $g$, into the extension (where the lift is determined by requiring that the lift is in $\mathbb{S}(1)$ ), and then repeat the preceding calculation in the extension. To do this we replace $a(g)^{h_{1}}$ by $\left|\sigma_{0}\right|(g)^{h_{0}}\left|\sigma_{1}\right|(g)^{h_{1}}$, and similarly for $h^{-1}$ (and recall that we have explicit product formulas for the functions $\left|\sigma_{j}\right|(g)$, and similarly for $\left.h\right)$. The torus-valued loop $e^{\left(\chi-\chi^{*}\right) h_{1}}$ has a vanishing diagonal term in its triangular factorization. However, a well-known formula for Toeplitz determinants implies that

$$
\left|\sigma_{j}\right|\left(e^{\left(\chi-\chi^{*}\right) h_{1}}\right)=\exp \left(-2 \sum_{j=1} j\left|\chi_{j}\right|^{2}\right) .
$$


Thus the lift of this torus-valued loop into the extension has diagonal term

$$
\exp \left(-2 \sum_{j=1} j\left|\chi_{j}\right|^{2}\left(h_{0}+h_{1}\right)\right)
$$

It is now straightforward to repeat the calculation above and conclude, as we did at the level of loops, that (i) the product of these lifts is in $\mathbb{S}(1)$ and (ii) the diagonal term of the product is the product of the diagonal terms of the factors. This implies the formulas in part (c).

To prove that the mapping is injective, recall that

$$
\begin{aligned}
& l\left(h^{-1} e^{\left(\chi-\chi^{*}\right) h_{1}} g\right)=l\left(h^{-1}\right) e^{-\chi^{*} h_{1}} a(h)^{h_{1}}\left(\begin{array}{cc}
1 & P_{-}\left(y^{*} e^{2 \chi^{*}}+x e^{2 \chi}\right) \\
0 & 1
\end{array}\right) a(h)^{-h_{1}} \\
& =\left(\begin{array}{cc}
1+\alpha^{*} & \gamma^{*} \\
\beta^{*} & 1+\delta^{*}
\end{array}\right) e^{-\chi^{*} h_{1}}\left(\begin{array}{cc}
1 & a(h)^{2} P_{-}\left(y^{*} e^{2 \chi^{*}}+x e^{2 \chi}\right) \\
0 & 1
\end{array}\right) \\
& =\left(\begin{array}{cc}
\left(1+\alpha^{*}\right) e^{-\chi^{*}} & \left(1+\alpha^{*}\right) e^{-\chi^{*}} a(h)^{2} P_{-}\left(y^{*} e^{2 \chi^{*}}+x e^{2 \chi}\right)+\gamma^{*} e^{\chi^{*}} \\
\beta^{*} e^{-\chi^{*}} & \beta^{*} e^{-\chi^{*}} a(h)^{2} P_{-}\left(y^{*} e^{2 \chi^{*}}+x e^{2 \chi}\right)+\left(1+\delta^{*}\right) e^{\chi^{*}}
\end{array}\right) .
\end{aligned}
$$

We need to show that this matrix determines $y$ (equivalently $h$ or $\eta$ ), $\chi$, and $x$ (or $g$ or $\zeta$ ).

By the form of the triangular factorization of $h$ in part (c) of Corollary 1, it is clear that the first row of $h$, evaluated at a point $z \in S^{1}$, is determined by $1+\alpha(z)$ and $\beta(z)$. Because $h(z) \in S U(2)$, this also determines $h(z)$. This means that the first row of $h$, as a holomorphic function of $z \in \Delta$ is determined up to a phase ambiguity by the ratio $\beta /(1+\alpha)$.

Now suppose that we are given (7.11). The first column determines the pair of holomorphic functions $\beta$ and $1+\alpha$ up to multiplication by a holomorphic function. This determines the ratio $\beta /(1+\alpha)$, and it also fixes the phase ambiguity. Thus the first column determines $h$. It is then clear that $\chi$ is determined. We can then use the $(1,2)$ entry to find $x$. This proves injectivity, and completes the proof of the Theorem.

\section{A Appendix}

In this appendix we will review some of the ideas in [7], relevant to this paper, from a slightly different perspective. The main rationale for including this appendix is that the basic arguments are valid in the more general Kac-Moody category. Throughout this appendix, we will use the notation and basic results in [6].

We start with the following data: $A$ is an irreducible symmetrizable generalized Cartan matrix; $\mathfrak{g}=\mathfrak{g}(A)$ is the corresponding Kac-Moody Lie algebra, realized via its standard (ChevalleySerre) presentation; $\mathfrak{g}=\mathfrak{n}^{-} \oplus \mathfrak{h} \oplus \mathfrak{n}^{+}$is the triangular decomposition; $\mathfrak{b}=\mathfrak{h} \oplus \mathfrak{n}^{+}$the upper Borel subalgebra; $G=G(A)$ is the algebraic group associated to $A$ by Kac-Peterson; $H, N^{ \pm}$and $B$ are the subgroups of $G$ corresponding to $\mathfrak{h}, \mathfrak{n}^{ \pm}$, and $\mathfrak{b}$, respectively; $K$ is the "unitary form" of $G ; T=K \cap H$ the maximal torus; and $W=N_{K}(T) / T \simeq N_{G}(H) / H$ is the Weyl group.

A basic fact is that $\left(G, B, N_{G}(H)\right)$ with Weyl group $W$ is an abstract Tits system. This yields a complete determination of all the (parabolic) subgroups between $B$ and $G$. They are described as follows.

Let $\Phi$ be a fixed subset of the simple roots. The subgroup of $W$ generated by the simple reflections corresponding to roots in $\Phi$ will be denoted by $W(\Phi)$. The parabolic subgroup corresponding to $\Phi, P=P(\Phi)$, is given by $P=B W(\Phi) B$. Given $\mathbf{w} \in N_{K}(T)$, we will denote its image in $W / W(\Phi)$ by $w$. 
The basic structural features of $G / P$ which we will need are the Birkhoff and Bruhat decompositions

$$
\begin{array}{ll}
G / P=\bigsqcup \Sigma_{w}, & \Sigma_{w}=N^{-} \mathbf{w} P, \\
G / P=\bigsqcup C_{w}, & C_{w}=B \mathbf{w} P
\end{array}
$$

respectively, where the indexing set is $W / W(\Phi)$ in both cases. The strata $\Sigma_{w}$ are infinite dimensional if $\mathfrak{g}$ is infinite dimensional, while the cells $C_{w}$ are always finite dimensional. Our initial interest is in the Schubert variety $\bar{C}_{w}$, the closure of the cell.

Fix $w \in W / W(\Phi)$. We choose a representative $\mathbf{w} \in N_{K}(T)$ of minimal length $n$, and we fix a factorization

$$
\mathbf{w}=r_{n} \cdots r_{1}
$$

where $r_{j}=i_{\gamma_{i}}\left(\begin{array}{ll}0 & i \\ i & 0\end{array}\right)$, and $i_{\gamma_{j}}: S L_{2} \rightarrow G$ is the canonical homomorphism of $S L_{2}$ onto the root subgroup corresponding to the simple root $\gamma_{j}$.

Proposition 4. For $\mathbf{w}$ as in (A.1), the map

$$
r_{n} \exp \left(\mathfrak{g}_{-\gamma_{n}}\right) \times \cdots \times r_{1} \exp \left(\mathfrak{g}_{-\gamma_{1}}\right) \rightarrow G / P:\left(p_{j}\right) \rightarrow p_{n} \cdots p_{1} P
$$

is a complex analytic isomorphism onto $C_{w}$.

This result is essentially (5) of [6] together with Tits's theory. We will include a proof for completeness.

Proof. Let $\Delta^{+}$denote the positive roots, $\Delta^{+}(\Phi)$ the positive roots which are combinations of elements from $\Phi$. The "Lie algebra of $P^{\prime}$ " is $\mathfrak{p}=\Sigma \mathfrak{g}_{-\beta} \oplus \mathfrak{b}$ where the sum is over $\beta \in \Delta^{+}(\Phi)$; this is the Lie algebra of $P$ in the sense that it is the subalgebra generated by the root spaces $\mathfrak{g}_{\gamma}$ for which exp $: \mathfrak{g}_{\gamma} \rightarrow G$ is defined and have image contained in $P$. The subgroups $\exp \left(\mathfrak{g}_{\gamma}\right)$ generate $P$. We also let $\mathfrak{p}^{-}$denote the subalgebra opposite $\mathfrak{p}: \mathfrak{p}^{-}=\sum \mathfrak{g}_{-\gamma}$, where the sum is over $\gamma \in \Delta^{+} \backslash \Delta^{+}(\Phi)$. The corresponding group will be denoted by $P^{-}$.

The cell $C_{w}$ is the image of the map $N^{+} \rightarrow G / P: u \rightarrow u \mathbf{w} P$. The stability subgroup at $\mathbf{w} P$ is $N^{+} \cap \mathbf{w} P \mathbf{w}^{-1}$.

At the Lie algebra level we have the splitting

$$
\mathfrak{n}^{+}=\mathfrak{n}^{+} \cap \operatorname{Ad}(\mathbf{w})(\mathfrak{p}) \oplus \mathfrak{n}^{+} \cap \operatorname{Ad}(\mathbf{w})\left(\mathfrak{p}^{-}\right) .
$$

The second summand equals $\mathfrak{n}_{w}^{+}=\oplus \mathfrak{g}_{\beta}$, where the sum is over roots $\beta>0$ with $w^{-1} \beta \in$ $-\left(\Delta^{+} \backslash \Delta^{+}(\Phi)\right)$. These roots $\beta$ are necessarily real, so that $\exp : \mathfrak{n}_{w}^{+} \rightarrow N_{w}^{+} \subseteq N^{+}$is welldefined.

For $q \in \mathbb{Z}^{+}$let $N_{q}^{+}$denote the subgroup corresponding to $\mathfrak{n}_{q}^{+}=\operatorname{span}\left\{\mathfrak{g}_{\beta}: \operatorname{height}(\beta) \geq q\right\}$. Then $N^{+} / N_{q}^{+}$is a finite dimensional nilpotent Lie group, and it is also simply connected. By taking $q$ sufficiently large and considering the splitting (A.2) modulo $\mathfrak{n}_{q}^{+}$, we conclude by finite dimensional considerations that each element in $N^{+}$has a unique factorization $n=n_{1} n_{2}$, where $n_{1} \in N_{w}^{+}$and $n_{2} \in N^{+} \cap \mathbf{w} P \mathbf{w}^{-1}$ :

$$
N^{+} \simeq N_{w}^{+} \times\left(N^{+} \cap \mathbf{w} P \mathbf{w}^{-1}\right) .
$$

The important point is that modulo $N_{q}^{+}$, we can control $N^{+} \cap \mathbf{w} P \mathbf{w}^{-1}$ by the exponential map.

The following lemma is standard. 
Lemma 5. In terms of the minimal factorization $\mathbf{w}=r_{n} \cdots r_{1}$, the roots $\beta>0$ with $\mathbf{w}^{-1} \beta<0$ are given by

$$
\beta_{j}=r_{n} \cdots r_{j+1}\left(\gamma_{j}\right)=r_{n} \cdots r_{j}\left(-\gamma_{j}\right), \quad 1 \leq j \leq n .
$$

Because $\mathbf{w}$ is a representative of $w \in W / W(\Phi)$ of minimal length, all of these $\beta_{j}$ satisfy $\mathbf{w}^{-1} \beta_{j} \in-\left(\Delta^{+} \backslash \Delta^{+}(\Phi)\right)$. Otherwise, if say $\mathbf{w}^{-1} \beta_{j} \in-\Delta^{+}(\Phi)$, then

$$
\mathbf{w}^{-1} r_{\beta_{j}} \mathbf{w}=r_{1} \cdots r_{j-1} r_{j} r_{j-1} \cdots r_{1} \in N_{K}(T) \cap P
$$

and $\mathbf{w}^{\prime}=\mathbf{w}\left(\mathbf{w}^{-1} r_{\beta_{j}} \mathbf{w}\right)=r_{n} \cdots \widehat{r}_{j} \cdots r_{1}$ would be a representative of $w$ of length $<n$ (here we have used the fact that $W(\Phi)=N_{K}(T) \cap P / T$, which follows from the Bruhat decomposition). For future reference we note this proves that

$$
N_{w}^{+}=N^{+} \cap\left(N^{-}\right)^{w}=N^{+} \cap\left(P^{-}\right)^{w}
$$

and (A.3) shows that

$$
N_{w}^{+} \times w \cong C_{w}
$$

Now for any $1 \leq p \leq q \leq n, \bigoplus_{p \leq j \leq p} \mathfrak{g}_{\beta_{j}}$ is a subalgebra of $\mathfrak{n}_{w}^{+}$. Thus by (A.3)

$$
\exp \left(\mathfrak{g}_{\beta_{n}}\right) \times \cdots \times \exp \left(\mathfrak{g}_{\beta_{1}}\right) \times w \cong C_{w} .
$$

This completes the proof of Proposition 4, when we write

$$
\exp \left(\mathfrak{g}_{\beta_{j}}\right)=r_{n} \cdots r_{j} \exp \left(\mathfrak{g}_{\gamma_{j}}\right) r_{j} \cdots r_{n}
$$

For each $j$, let $P_{j}$ denote the parabolic subgroup $i_{\gamma_{j}}\left(S L_{2}\right) B$. Let

$$
\gamma_{\mathbf{w}}=P_{n} \times_{B} \cdots \times_{B} P_{1} / B
$$

where

$$
P_{n} \times \cdots \times P_{1} \times B \times \cdots \times B \rightarrow P_{n} \times \cdots \times P_{1}
$$

is given by

$$
\left(p_{j}\right) \times\left(b_{j}\right) \rightarrow\left(p_{n} b_{n}, b_{n}^{-1} p_{n-1} b_{n-1}, \ldots, b_{2}^{-1} p_{1} b_{1}\right) .
$$

We have written " $\gamma_{\mathbf{w}}$ " instead of " $\gamma_{w}$ " to indicate that this compact complex manifold depends upon the factorization (A.1).

Corollary 2. The map

$$
\gamma_{\mathbf{w}} \rightarrow \bar{C}_{w}:\left(p_{j}\right) \rightarrow p_{n} \cdots p_{1} P
$$

is a (Bott-Samelson) desingularization of $\bar{C}_{w}$.

This is an immediate consequence of Proposition 4.

Let

$$
S L_{2}^{\prime}=\left\{g=\left(\begin{array}{ll}
a & b \\
c & d
\end{array}\right) \in S L(2, \mathbb{C}): a \neq 0\right\} .
$$


Corollary 3. Consider the surjective map

$$
S L_{2} \times \cdots \times S L_{2} \rightarrow \bar{C}_{w}:\left(g_{j}\right) \rightarrow r_{n} i_{\gamma_{n}}\left(g_{n}\right) \cdots r_{1} i_{\gamma_{1}}\left(g_{1}\right) P .
$$

The inverse image of $C_{w}$ is $S L_{2}^{\prime} \times \cdots \times S L_{2}^{\prime}$.

Proof. Let $\sigma=r_{n-1} \cdots r_{1}$. It suffices to show that for the natural actions

$$
\begin{aligned}
& r_{n} i_{\gamma_{n}}\left(S L_{2}^{\prime}\right) \times C_{\bar{\sigma}} \rightarrow C_{w}, \\
& r_{n} i_{\gamma_{n}}\left(S L_{2} \backslash S L_{2}^{\prime}\right) \times C_{\bar{\sigma}} \rightarrow \bar{C}_{w},
\end{aligned}
$$

and

$$
r_{n} i_{\gamma_{n}}\left(S L_{2}\right) \times\left(\bar{C}_{\bar{\sigma}} \backslash C_{\bar{\sigma}}\right) \rightarrow \bar{C}_{w} \backslash C_{w}
$$

The first line, (A.6), follows from Proposition 4, since $i_{\gamma_{n}}\left(S L_{2}^{\prime}\right) \subseteq \exp \left(-\mathfrak{g}_{-\gamma_{n}}\right) B$ and $B \times C_{\bar{\sigma}} \subseteq C_{\bar{\sigma}}$. The second line follows from

$$
r_{n} i_{\gamma_{n}}\left(\begin{array}{ll}
0 & b \\
c & d
\end{array}\right) \cdot C_{\bar{\sigma}}=i_{\gamma_{n}}\left(\begin{array}{cc}
c & b \\
0 & d
\end{array}\right) \cdot C_{\bar{\sigma}} \subseteq C_{\bar{\sigma}}
$$

For the third line it's clear that the image of the left hand side is a union of cells, since we can replace $r_{n} i_{\gamma_{n}}\left(S L_{2}\right)$ by $P_{n}$. This image is at most $n-1$ dimensional. Therefore it must have null intersection with $C_{w}$.

\section{Corollary 4.}

(a) Let $k(\zeta)$ be defined as in (5.3). The map

$$
\mathbb{C}^{n} \rightarrow C_{w}:\left(\zeta_{n}, \ldots, \zeta_{1}\right) \rightarrow r_{n} i_{\gamma_{n}}\left(k\left(\zeta_{n}\right)\right) \cdots r_{1} i_{\gamma_{1}}\left(k\left(\zeta_{1}\right)\right) P
$$

is a real analytic isomorphism.

(b) In terms of the parameterization in (a), and the parameterization of $C_{w}$ by $N^{+} \cap \mathbf{w} P \mathbf{w}^{-1}$ (see (A.4) and (A.5)), Haar measure (unique up to a constant) is given by

$$
\begin{aligned}
d \lambda_{N^{+} \cap w P w^{-1}} & =\prod_{j=1}^{n}\left(1+\left|\zeta_{j}\right|^{2}\right)^{\delta\left(w_{j-1}^{-1} h_{\gamma_{j}} w_{j-1}\right)-1} \\
& =\prod_{1 \leq i<j \leq n}\left(1+\left|\zeta_{j}\right|^{2}\right)^{-\gamma_{i}\left(w_{i-1} w_{j-1}^{-1} h_{\gamma_{j}} w_{j-1} w_{i-1}^{-1}\right)},
\end{aligned}
$$

where $\delta=\sum \Lambda_{j}$, the sum of the dominant integral functionals for $\mathfrak{g}$.

Proof. The proof of (a) is by induction on $n$. We write $w_{n}$ in place of $w$, and $k\left(\zeta_{n}\right)=$ $l\left(\zeta_{n}\right) a\left(\zeta_{n}\right) u\left(\zeta_{n}\right)$ for its $S L(2, \mathbb{C})$ triangular factorization.

The case $n=1$ is obvious. Assume the result holds for $n-1$. Suppose that

$$
r_{n} i_{\gamma_{n}}\left(k\left(\zeta_{n}\right)\right) \cdots r_{1} i_{\gamma_{1}}\left(k\left(\zeta_{1}\right)\right) P=r_{n} i_{\gamma_{n}}\left(k\left(\zeta_{n}^{\prime}\right)\right) \cdots r_{1} i_{\gamma_{1}}\left(k\left(\zeta_{1}^{\prime}\right)\right) P .
$$

Since $C_{w_{n-1}}$ is $B^{+}$-stable, this equation implies

$$
r_{n} i_{\gamma_{n}}\left(l\left(\zeta_{n}\right)\right) x P=r_{n} i_{\gamma_{n}}\left(l\left(\zeta_{n}^{\prime}\right)\right) y P,
$$

where $x P, y P \in C_{w_{n-1}}$. Proposition 4 implies that $\zeta_{n}=\zeta_{n}^{\prime}$. Given this, the induction hypothesis implies that $\zeta_{j}=\zeta_{j}^{\prime}$ for $j<n$. Thus the map is injective. 
Since $k\left(\zeta_{n}\right)=l\left(\zeta_{n}\right)$ modulo $B^{+}$, and elements of $B^{+}$stabilize $C_{w_{n-1}}$, Proposition 4 also implies the map is surjective. This proves (a).

Now consider (b). We will first establish the second formula by induction. We will then show the two formulas are equivalent.

Recall the factorization

$$
i_{\gamma}(k(\zeta))=i_{\gamma}\left(\left(\begin{array}{ll}
1 & 0 \\
\zeta & 1
\end{array}\right)\left(\begin{array}{cc}
a(\zeta) & 0 \\
0 & a(\zeta)^{-1}
\end{array}\right)\left(\begin{array}{cc}
1 & -\bar{\zeta} \\
0 & 1
\end{array}\right)\right)
$$

From this the case $n=1$ is clear. Now suppose the second formula in (b) is valid for $n-1$ and $n>1$. We have

$$
r_{n} i_{\gamma_{n}}\left(k\left(\zeta_{n}\right)\right)=r_{n} i_{\gamma_{n}}\left(\left(\begin{array}{cc}
1 & 0 \\
\zeta_{n} & 1
\end{array}\right)\left(\begin{array}{cc}
a\left(\zeta_{n}\right) & 0 \\
0 & a\left(\zeta_{n}\right)^{-1}
\end{array}\right)\left(\begin{array}{cc}
1 & -\bar{\zeta}_{n} \\
0 & 1
\end{array}\right)\right)
$$

The action of

$$
i_{\gamma_{n}}\left(\left(\begin{array}{cc}
1 & -\bar{\zeta}_{n} \\
0 & 1
\end{array}\right)\right)
$$

on $C_{w_{n-1}}$, in terms of the parameterization by $N^{+} \cap w_{n-1} N^{-} w_{n-1}^{-1}$, is by translation. So this action preserves Lebesgue measure. The action of

$$
i_{\gamma_{n}}\left(\left(\begin{array}{cc}
a\left(\zeta_{n}\right) & 0 \\
0 & a\left(\zeta_{n}\right)^{-1}
\end{array}\right)\right)
$$

on $C_{w_{n-1}}$, in terms of the parameterization by $N^{+} \cap w_{n-1} N^{-} w_{n-1}^{-1}$, is by conjugation, and we can easily calculate the effect on volume. In a routine way this leads to the second formula in (b).

To prove the formulas in (b) are equivalent, recall that for a simple positive root $\gamma$, with corresponding reflection $r_{\gamma}$, one has

$$
r_{\gamma} \delta=\delta-\gamma
$$

This implies that

$$
\begin{aligned}
\left(w_{j-1} \delta\right)\left(h_{\gamma_{j}}\right)-1 & =\left(r_{j-1} \cdots r_{1} \delta\right)\left(h_{\gamma_{j}}\right)-1=\left(r_{j-1} \cdots r_{2}\left(\delta-\gamma_{1}\right)\right)\left(h_{\gamma_{j}}\right)-1 \\
& =\left(\delta-\gamma_{j-1}-r_{j-1} \gamma_{j-2}-\cdots-r_{j-1} \cdots r_{2} \gamma_{1}\right)\left(h_{\gamma_{j}}\right)-1 \\
& =-\sum_{i=1}^{j-1}\left(r_{j-1} \cdots r_{i+1} \gamma_{i}\right)\left(h_{\gamma_{j}}\right)=-\sum_{i=1}^{j-1}\left(w_{j-1} w_{i-1}^{-1} \gamma_{i}\right)\left(h_{\gamma_{j}}\right) .
\end{aligned}
$$

This completes the proof of (b).

Fix an integral functional $\lambda \in \mathfrak{h}^{*}$ which is antidominant. Denote the (algebraic) lowest weight module corresponding to $\lambda$ by $L(\lambda)$, and a lowest weight vector by $\sigma_{\lambda}$. Let $\Phi$ denote the simple roots $\gamma$ for which $\lambda\left(h_{\gamma}\right)=0$, where $h_{\gamma}$ is the coroot, $P=P(\Phi)$ the corresponding parabolic subgroup. The Borel-Weil theorem in this context realizes $L(\lambda)$ as the space of strongly regular functions on $G$ satisfying

$$
f(g p)=f(g) \lambda(p)^{-1}
$$

for all $g \in G$ and $p \in P$, where we have implicitly identified $\lambda$ with the character of $P$ given by

$$
\lambda\left(u_{1} w \exp (x) u_{2}\right)=\exp \lambda(x)
$$


for $x \in \mathfrak{h}, u_{1}, u_{2} \in N^{+}, w \in W(\Phi)$. Thus we can view $L(\lambda)$ as a space of sections of the line bundle

$$
\mathcal{L}_{\lambda}=G \times_{\lambda} \mathbb{C} \rightarrow G / P .
$$

If $\mathfrak{g}$ is of finite type, then $L(\lambda)=H^{0}\left(\mathcal{L}_{\lambda}\right)$; if $\mathfrak{g}$ is affine (and untwisted), then $L(\lambda)$ consists of the holomorphic sections of finite energy, as in [11].

Normalize $\sigma_{\lambda}$ by $\sigma_{\lambda}(1)=1$.

Proposition 5. Let $w \in W / W(\Phi)$, and let $w=r_{n} \cdots r_{1}$ be a representative of minimal length $n$. Let $w_{j}=r_{j} \cdots r_{1}$. The positive roots mapped to negative roots by $w$ are given by

$$
\tau_{j}=w_{j-1}^{-1}\left(\gamma_{j}\right), \quad 1 \leq j \leq n ;
$$

let $\lambda_{j}=-\lambda\left(h_{\tau_{j}}\right)$, where $h_{\tau}$ is the coroot corresponding to $\tau$. Then

$$
\sigma_{\lambda}^{w}\left(r_{n} i_{\gamma_{n}}\left(g_{n}\right) \cdots r_{1} i_{\gamma_{1}}\left(g_{1}\right)\right)=\sigma_{\lambda}\left(w_{n-1}^{-1} i_{\gamma_{n}}\left(g_{n}\right) w_{n-1} \cdots w_{1}^{-1} i_{\gamma_{2}}\left(g_{2}\right) w_{1} i_{\gamma_{1}}\left(g_{1}\right)\right)=\prod_{1}^{n} a_{j}^{\lambda_{j}},
$$

where $g=\left(\begin{array}{ll}a & b \\ c & d\end{array}\right) \in S L_{2}$.

Proof of Proposition 5. The claim about the $\tau_{j}$ follows from Lemma 5. None of these roots lie in $\Delta^{+}(\Phi)$, by the same argument as follows (5). Thus each $\lambda_{j}>0$. It follows that $\Pi a_{j}^{\lambda_{j}}$ is nonzero precisely on the set $S L_{2}^{\prime} \times \cdots \times S L_{2}^{\prime}$.

Now $\sigma_{\lambda}^{w}$, viewed as a section of $\mathcal{L}_{\lambda} \rightarrow G / P$, is nonzero precisely on the $w$-translate of the largest stratum,

$$
w \Sigma_{1}=w P^{-} P=\left(P^{-}\right)^{w} w P .
$$

We claim the intersection of this with $\bar{C}_{w}$ is $C_{w}$. In one direction

$$
C_{w}=\left(N^{+} \cap\left(P^{-}\right)^{w}\right) w P \subseteq\left(P^{-}\right)^{w} w P
$$

by (A.4). On the other hand $\left(N^{+} \cap\left(P^{-}\right)^{w}\right)$ is a closed finite dimensional subgroup of $\left(P^{-}\right)^{w}$. Since $\left(P^{-}\right)^{w}$ is topologically equivalent to $w \Sigma_{1}$, the limit points of $C_{w}$ must be in the complement of $w \Sigma_{1}$. This establishes the other direction.

It now follows from Proposition 4 that $\sigma_{\lambda}^{w}$ is also nonzero precisely on $S L_{2}^{\prime} \times \cdots \times S L_{2}^{\prime}$, viewed as a function of $\left(g_{n}, \ldots, g_{1}\right)$.

Write

$$
\begin{aligned}
& \sigma_{\lambda}\left(w_{n-1}^{-1} i_{\gamma_{n}}\left(g_{n}\right) w_{n-1} w_{n-2}^{-1} i_{\gamma_{n-1}}\left(g_{n-1}\right) w_{n-2} \cdots i_{\gamma_{1}}\left(g_{1}\right)\right) \\
& =\sigma_{\lambda}\left(i_{\tau_{n}}\left(g_{n}\right) i_{\tau_{n-1}}\left(g_{n-1}\right) \cdots i_{\tau_{1}}\left(g_{1}\right)\right)
\end{aligned}
$$

where $i_{\tau_{i}}(\cdot)=w_{i-1}^{-1} i_{\gamma_{i}}(\cdot) w_{i-1}$. Because

$$
w_{i-1}^{-1}\left(\gamma_{i}\right)>0,
$$

$i_{\tau_{j}}: S L_{2} \rightarrow G$ is a homomorphism onto the root subgroup corresponding to $\tau_{j}$ which is compatible with the canonical triangular decompositions.

$$
\begin{aligned}
\text { For } g & =\left(\begin{array}{ll}
a & b \\
c & d
\end{array}\right) \in S L_{2}^{\prime}, \text { write } g=L D U, \text { where } \\
& L=\left(\begin{array}{cc}
1 & 0 \\
c a^{-1} & 1
\end{array}\right), \quad D=\left(\begin{array}{cc}
a & 0 \\
0 & a^{-1}
\end{array}\right), \quad U=\left(\begin{array}{cc}
1 & a^{-1} b \\
0 & 1
\end{array}\right) .
\end{aligned}
$$


Then for $\left(g_{j}\right) \in S L_{2}^{\prime} \times \cdots \times S L_{2}^{\prime},($ A.7) equals

$$
\begin{aligned}
& \sigma_{\lambda}\left(i_{\tau_{n}}\left(L_{n} D_{n} U_{n}\right) \cdots i_{\tau_{1}}\left(L_{1} D_{1} U_{1}\right)\right) \\
& \quad=\sigma_{\lambda}\left(i_{\tau_{n}}\left(L_{n} U_{n}^{\prime}\right) i_{\tau_{n-1}}\left(L_{n-1}^{\prime} U_{n-1}^{\prime}\right) \cdots i_{\tau_{1}}\left(L_{1}^{\prime} U_{1}^{\prime}\right) i_{\tau_{n}}\left(D_{n}\right) \cdots i_{\tau_{1}}\left(D_{1}\right)\right) \\
& \quad=\sigma_{\lambda}\left(i_{\tau_{n}}\left(L_{n} U_{n}^{\prime}\right) \cdots i_{\tau_{1}}\left(L_{1}^{\prime} U_{1}^{\prime}\right)\right) \Pi a_{j}^{\lambda_{j}}
\end{aligned}
$$

where each $L_{j}^{\prime}\left(U_{j}^{\prime}\right)$ has the same form as $L_{j}\left(U_{j}\right.$, respectively). This follows from the fact that $H$ normalizes each $\exp \left(\mathfrak{g}_{ \pm r}\right)$.

Now each $L_{j}^{\prime} U_{j}^{\prime} \in S L_{2}^{\prime}$, so that $i_{\tau_{n}}\left(L_{n} U_{n}^{\prime}\right) \cdots i_{\tau_{1}}\left(L_{1}^{\prime} U_{1}^{\prime}\right)$ is in $\Sigma_{1}$. We now conclude that

$$
\sigma_{\lambda}\left(i_{\tau_{n}}\left(L_{n} U_{n}^{\prime}\right) \cdots i_{\tau_{1}}\left(L_{1}^{\prime} U_{1}^{\prime}\right)\right)=1
$$

by the fundamental theorem of algebra, since this is polynomial and never vanishes.

\section{References}

[1] Brezis H., New questions related to topological degree, in The Unity of Mathematics, Prog. Math., Vol. 244, Birkhäuser, Boston, MA, 2006, 137-154.

[2] Caine A., Compact symmetric spaces, triangular factorization, and Poisson geometry, J. Lie Theory 18 (2008), 273-294, math.SG/0608454.

[3] Caine A., Pickrell D., Homogeneous Poisson structures on symmetric spaces, Int. Math. Res. Not., to appear, arXiv:0710.4484.

[4] Evens S., Lu J.-H., On the variety of Lagrangian subalgebras. I, Ann. Sci. École Norm. Sup. (4) 34 (2001), 631-668, math.DG/9909005.

[5] Kac V., Infinite-dimensional Lie algebras. An introduction, Birkhäuser, Boston, MA, 1983.

[6] Kac V., Constructing groups from infinite-dimensional Lie algebras, in Infinite-Dimensional Groups with Applications (Berkeley, Calif., 1984), Editor V. Kac, Math. Sci. Res. Inst. Publ., Vol. 4, Springer, New York, 1985, 167-216.

[7] Lu J.-H., Coordinates on Schubert cells, Kostant's harmonic forms, and the Bruhat-Poisson structure on G/B, Transform. Groups 4 (1999), 355-374, dg-ga/9610009.

[8] Odzijewicz A., Ratiu T., Banach Lie-Poisson spaces and reduction, Comm. Math. Phys. 243 (2003), 1-54, math.SG/0210207.

[9] Pickrell D., An invariant measure for the loop space of a simply connected compact symmetric space, J. Funct. Anal. 234 (2006), 321-363, math-ph/0409013.

[10] Pickrell D., A survey of conformally invariant measures on $H^{m}(\Delta)$, math.PR/0702672.

[11] Pressley A., Segal G., Loop groups, Oxford Mathematical Monographs, Oxford Science Publications, The Clarendon Press, Oxford University Press, New York, 1986.

[12] Widom H., Asymptotic behavior of block Toeplitz matrices and determinants. II, Adv. Math. 21 (1976), $1-29$. 